\title{
Human body epigenome maps reveal noncanonical DNA methylation variation
}

\author{
Matthew D. Schultz ${ }^{1,2} \dagger^{*}$, Yupeng He ${ }^{1,2}$, John W. Whitaker ${ }^{\dagger}$, Manoj Hariharan ${ }^{2}$, Eran A. Mukamel ${ }^{4,5}$, Danny Leung ${ }^{6}$, \\ Nisha Rajagopal ${ }^{6}$, Joseph R. Nery ${ }^{2}$, Mark A. Urich ${ }^{2}$, Huaming Chen ${ }^{2}$, Shin Lin ${ }^{7}$, Yiing Lin ${ }^{8}$, Inkyung Jung ${ }^{6}$, Anthony D. Schmitt ${ }^{6}$, \\ Siddarth Selvaraj ${ }^{1}$, Bing Ren ${ }^{6,9}$, Terrence J. Sejnowski ${ }^{4,10,11}$, Wei Wang ${ }^{3,12}$ \& Joseph R. Ecker ${ }^{2,11}$
}

Understanding the diversity of human tissues is fundamental to disease and requires linking genetic information, which is identical in most of an individual's cells, with epigenetic mechanisms that could have tissue-specific roles. Surveys of DNA methylation in human tissues have established a complex landscape including both tissue-specific and invariant methylation patterns ${ }^{1,2}$. Here we report high coverage methylomes that catalogue cytosine methylation in all contexts for the major human organ systems, integrated with matched transcriptomes and genomic sequence. By combining these diverse data types with each individuals' phased genome ${ }^{3}$, we identified widespread tissue-specific differential CG methylation (mCG), partially methylated domains, allele-specific methylation and transcription, and the unexpected presence of non-CG methylation $(\mathrm{mCH})$ in almost all human tissues. $\mathrm{mCH}$ correlated with tissue-specific functions, and using this mark, we made novel predictions of genes that escape $\mathrm{X}$-chromosome inactivation in specific tissues. Overall, DNA methylation in several genomic contexts varies substantially among human tissues.

To understand the variability of DNA methylation across human tissues better, we obtained post-mortem samples of 18 tissue types from 4 individuals ( 5 singletons, 8 duplicates and 5 triplicates; Fig. 1a, Supplementary Methods and Supplementary Table 1) and performed deep transcriptome (36 messenger-RNA-seq samples; 120-475 million reads per sample), base-resolution methylome (36 MethylC-seq ${ }^{4}$ samples; $30-80 \times$ genome coverage per sample), and genome sequencing ( 4 whole genome sequences; $20-45 \times$ genome coverage per sample). We focused our initial analysis on cytosines in the CG context and used a previously published method ${ }^{2}$ to identify differential methylation (Supplementary Methods). We found that $15.4 \%(4,073,896$ out of $26,474,560$ sites tested) of CG sites in these experiments are strongly differentially methylated (minimum methylation difference $\geq 0.3$; Extended Data Fig. 1a), which is similar to a previous study ${ }^{2}$. To identify differentially methylated regions (DMRs), we combined sites within 500 base pairs (bp) of one another and found $1,198,132$ DMRs. Even with these stringent criteria, 719,837 (60.1\%) of the DMRs we identified were novel ${ }^{2,5}$.

As expected, hypomethylation at DMRs correlated with tissuespecific functions ${ }^{2,6}$. For example, strongly hypomethylated DMRs in the aorta overlap with aorta-specific super enhancers ${ }^{7}$ around $M Y H 10$, a gene involved in blood vessel function ${ }^{8}$ (Fig. 1b). To validate our DMRs further, we performed hierarchical clustering on their weighted methylation levels ${ }^{9}$ (Supplementary Methods, Fig. 1c and Extended Data Fig. 1b, c). Tissues that were part of the same organ system clustered together (for example, heart and muscle tissues). We compared these results to a clustering of differentially expressed genes identified in the transcriptomes and found a similar separation of organ systems (Supplementary Methods, Fig. 1d and Extended Data Fig. 1d). Furthermore, Genomic Regions Enrichment of Annotations Tool $^{10}$ analysis on the most hypomethylated tissue-specific DMRs revealed many tissue-specific functions (Extended Data Fig. 1e, f, Supplementary Methods and Supplementary Tables 2-3).

To examine the relationship between methylation and transcription, we correlated the methylation levels of DMRs and the expression of the closest genes (Fig. 2a, Extended Data Fig. 2a, b and Supplementary Methods). As expected, methylation in DMRs had a negative correlation with expression, and this correlation grew stronger closer to the transcription start site. The strongest negative correlation was not in gene promoters but downstream of the promoter up to 8 kilobases $(\mathrm{kb})$ away (intragenic $(0.3 \mathrm{~kb}$ to $8 \mathrm{~kb})$ versus promoter region and upstream region $(-2 \mathrm{~kb}$ to $0.3 \mathrm{~kb})$ median Spearman correlation coefficient difference -0.07 ; Mann-Whitney $P=4.2 \times 10^{-17}$; Fig. $2 \mathrm{a}$ ). This analysis shows that transcription is strongly associated with intragenic DMRs in the tissues we examined, extending similar observations in cancer methylomes ${ }^{11}$.

These intragenic methylation differences have previously been suggested to mark intragenic CG islands (CGIs) or CGI shores,12-14 However, only a small fraction of intragenic DMRs fell in these features (19\%; Extended Data Fig. 2c). In addition, predicted enhancers and putative promoters only accounted for $23 \%$ and $22 \%$ of intragenic DMRs, respectively, suggesting that the remaining DMRs, which we call undefined intragenic DMRs (uiDMRs), represent an unrecognized set of functional elements (35\%; Extended Data Fig. 2c and Supplementary Methods). The methylation level of these uiDMRs correlated strongly with the expression of the genes containing them. To examine their regulatory potential, we plotted their histone modification profiles (histone 3 Lys 4 methylation (H3K4me1), H3K4me3, H3K27ac, $\mathrm{H} 3 \mathrm{~K} 9 \mathrm{me} 3, \mathrm{H} 3 \mathrm{k} 27 \mathrm{me} 3$ and H3K36me3) derived from the same tissue samples ${ }^{15}$ and found five classes: weak enhancer, promoter-proximal, transcribed, poised enhancer and unmarked (Extended Data Figs $2 \mathrm{~d}-\mathrm{h}$, $3 \mathrm{a}, \mathrm{b}$ and Supplementary Methods). Classes with strong, active histone modifications were moderately negatively correlated with expression (weak enhancer and proximal promoter uiDMRs; median Spearman correlation coefficient -0.32 and -0.16 , respectively); whereas, uiDMRs with less active histone modifications exhibited a weak negative correlation (transcribed and poised enhancer uiDMRs). Notably, the correlation between expression and methylation at promoter-proximal

${ }^{1}$ Bioinformatics Program, University of California, San Diego, La Jolla, California 92093, USA. ${ }^{2}$ Genomic Analysis Laboratory, The Salk Institute for Biological Studies, La Jolla, California 92037, USA.

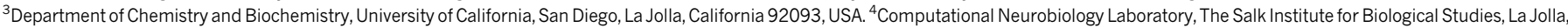
California 92037, USA. ${ }^{5}$ Department of Cognitive Science, University of California, San Diego, La Jolla, California 92037, USA. ${ }^{6}$ Ludwig Institute for Cancer Research, La Jolla, California 92093, USA.

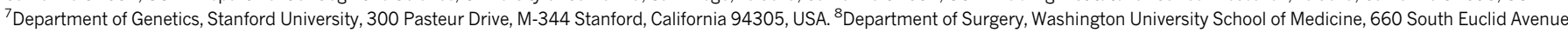

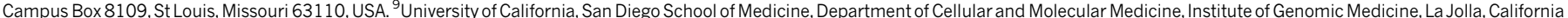

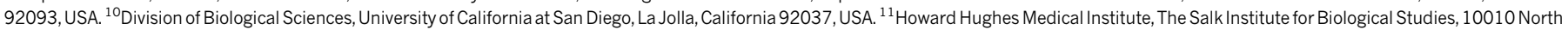

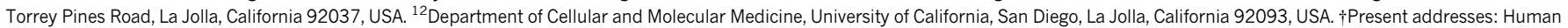
Longevity Inc., La Jolla, California 92121, USA (M.D.S.); Discovery Science, Janssen Pharmaceutical of Johnson \& Johnson, La Jolla, California 92121 , USA (J.W.W.).

*These authors contributed equally to this work. 

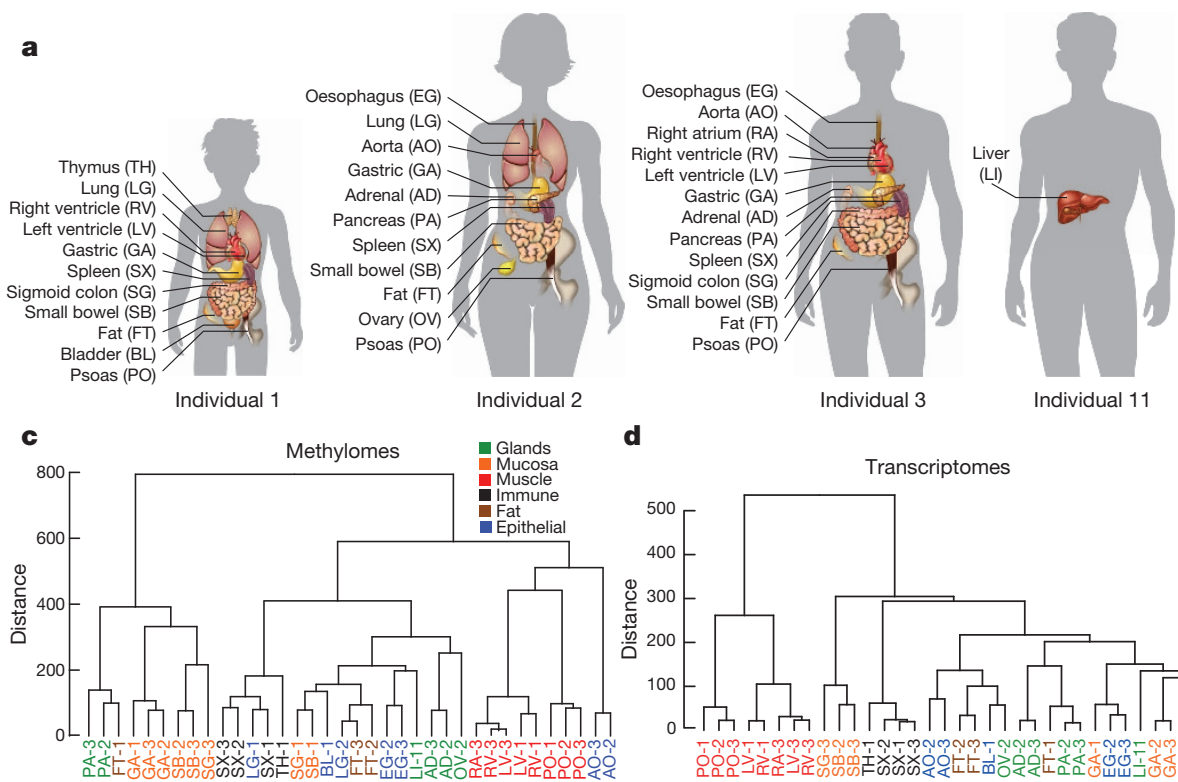

d
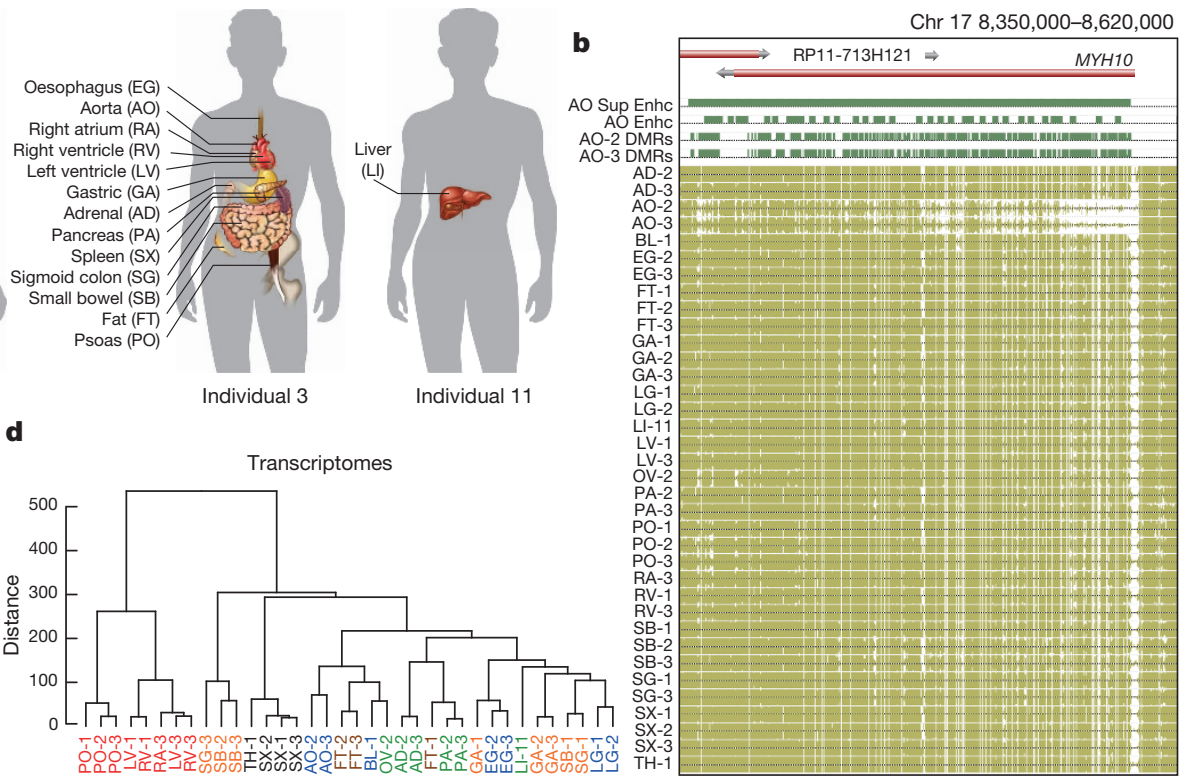

Figure $1 \mid$ The methylomes and transcriptomes of human tissues. a, The tissues analysed in this study. Samples are denoted by the two letter code in parentheses followed by an individual ID. b, Browser screenshot of an example DMR. The top track contains gene models. The following four tracks contain green blocks indicating the location of super enhancers, enhancers and hypomethylated DMRs in the aorta, respectively. The remaining tracks display methylation data from each sample. Gold ticks are CG sites with heights proportional to their methylation level. Ticks on the forward and reverse strand are projected upward and downward from the dotted line, respectively. c, d, Hierarchical clustering of DMR methylation levels (c) and expression levels of differentially expressed genes (d). Colours indicate the organ systems each sample belongs to.
uiDMRs was as strong as the correlation with intragenic DMRs that overlapped strong promoters (Extended Data Fig. 4 and Supplementary Methods), indicating that intragenic promoter and promoterproximal sequences are more predictive of changes in methylation than those enriched for enhancer-like chromatin modifications.

By contrast, unmarked uiDMRs showed a weakly positive correlation with expression (Extended Data Fig. 4d). Notably, we found many of the motifs enriched in tissue-specific uiDMRs were present in tissuespecific enhancers (for example, HNF4a (ref. 16) in liver-specific uiDMRs), suggesting that these DMRs are tissue-specific regulatory elements (Supplementary Methods and Supplementary Tables 4 and 5). Recently, hypomethylated regions that appear inactive in adult tissues but active during fetal development were identified in mice ${ }^{6}$. We examined the DNase I hypersensitivity profiles of unmarked uiDMRs in matched fetal tissues ${ }^{17}$ and found an enrichment of hypersensitivity (Extended Data Fig. 5 and Supplementary Table 6), suggesting that hypomethylation of inactive DMRs can be maintained at regions active earlier in development.

We next examined whether variation in methylation is associated with genetic variation across individuals, which has not been widely characterized in healthy primary tissues or using whole-genome bisulphite sequencing ${ }^{18,19}$.To identify individual-specific DMRs, we used a method ${ }^{20}$ that is sensitive to these differences unlike the methodology used above (Supplementary Methods). We first restricted our analysis to triplicated samples and ranked DMRs by a tissue-specific methylation outlier score that is largest when the methylation level in one individual differs from the other two. We found an $\sim 1$.6-fold enrichment of single nucleotide polymorphisms (SNPs) associating with methylation changes in the top 2,500 methylation-outlierscore-ranked DMRs in all tissues (Supplementary Methods). We then used the Epigram pipeline ${ }^{21}$ to predict tissue-specific methylation from DNA motifs in these DMRs and found them highly predictive (average area under the curve (AUC) 0.79; Supplementary Methods). These full models used an average of 156 motifs; however, an average AUC of 0.74 was achieved using only 20 core transcription factor motifs per tissue.
We then identified groups of corresponding motifs by clustering the sets of tissue-specific motifs (Supplementary Methods). The motif groups were clustered by their tissue hypo- and hypermethylation specificities (Fig. 2b). In total, 42 out of 95 motifs only had hypomethylation specificity; for example, MEIS, which is involved in heart development ${ }^{22}$, is hypomethylated in the left ventricle, right atrium and right ventricle. We also identified 34 motifs enriched at both hypomethylated DMRs in some tissues, and in hyper-methylated DMRs in some other tissues. Three of these motifs match transcription factor families (FOX, HOX and GATA) and are most significantly enriched in hypomethylated regions, suggesting that they are primarily involved in regulating hypomethylation.

Mammalian cells have high genome-wide levels of mCG, with the exception of a cultured human fetal fibroblast cell line (IMR90) ${ }^{4}$, cancer cells ${ }^{23,24}$ and placenta (PLA) ${ }^{25}$. Surprisingly, large regions of the pancreatic methylomes (PA-2 and PA-3) were significantly hypomethylated (Extended Data Fig. 6a). We developed a method to identify partially methylated domains (PMDs) genome-wide (Supplementary Tables 7-8 and Supplementary Methods) and found pancreatic PMDs were smaller than those in IMR90 and PLA (Extended Data Fig. 6b) and covered a smaller fraction of the genome (Fig. 2c). All pairs of PMDs overlapped significantly, indicating that these regions are largely shared ( $>40 \%$ overlap; $P<0.001$; Extended Data Fig. $6 c$ ).

Genes in samples with PMDs are transcriptionally repressed ${ }^{25,26}$, but these regions also show reduced expression in all of the tissues we surveyed whether or not a PMD is present (Fig. 2d). In both IMR90 and PA-2, these regions showed an enrichment in repressive modifications (H3K27me3 and $\mathrm{H} 3 \mathrm{~K} 9 \mathrm{me} 3$; median difference 0.025-0.168 reads per kilobase per million (RPKM); Mann-Whitney $P<2.51 \times 10^{-161}$ ) and a depletion in active modifications (H3K4me1, H3K27ac and H3K36me3; median difference 0.050-0.012 RPKM; Mann-Whitney $P<2.03 \times 10^{-53}$ ) compared to shuffled regions (Fig. 2e, f, Extended Data Fig. 6 d, e and Supplementary Methods), which provides a potential mechanism for their repression. To try to account for this global hypomethylation, we plotted the expression levels of DNMT1, DNMT3A, DNMT3B and DNMT3L but found no 


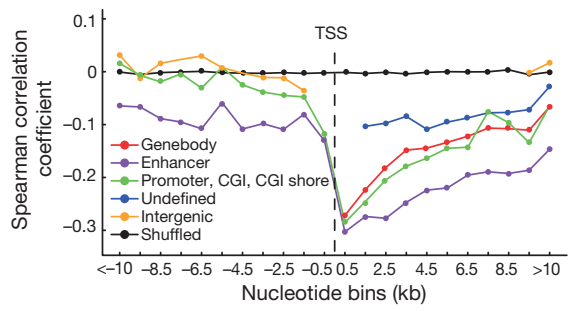

d

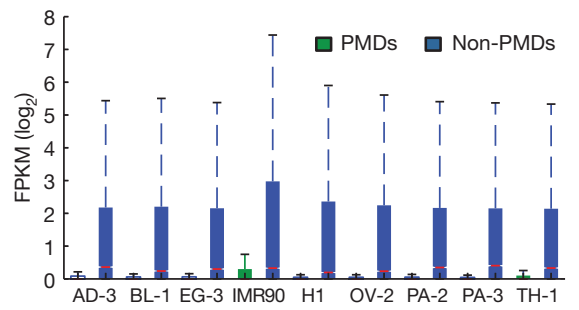

b

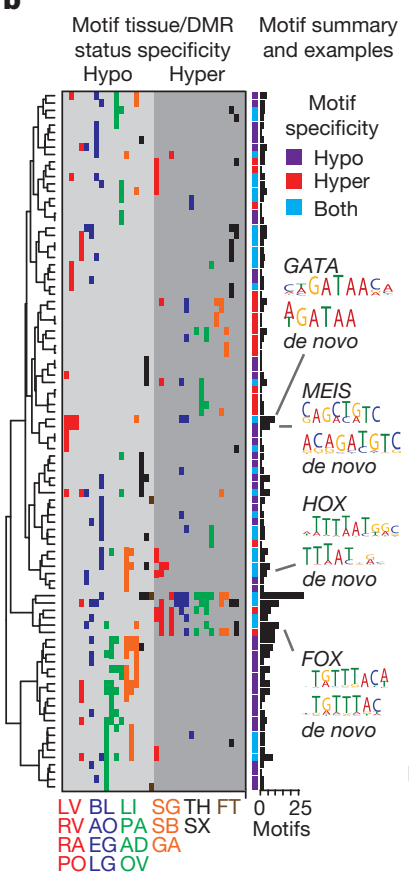

C

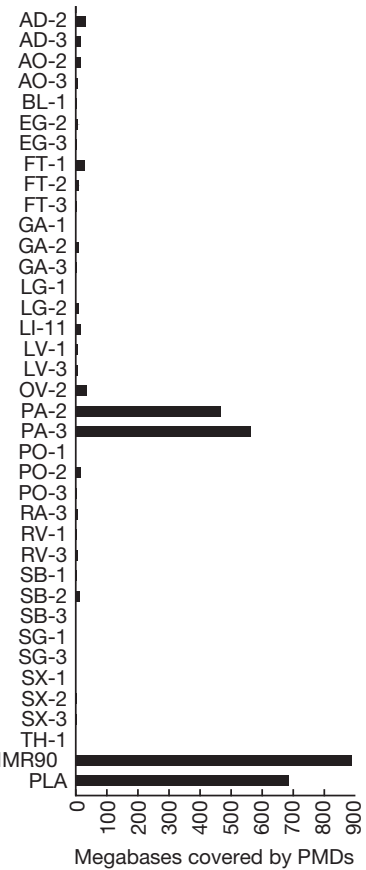

e
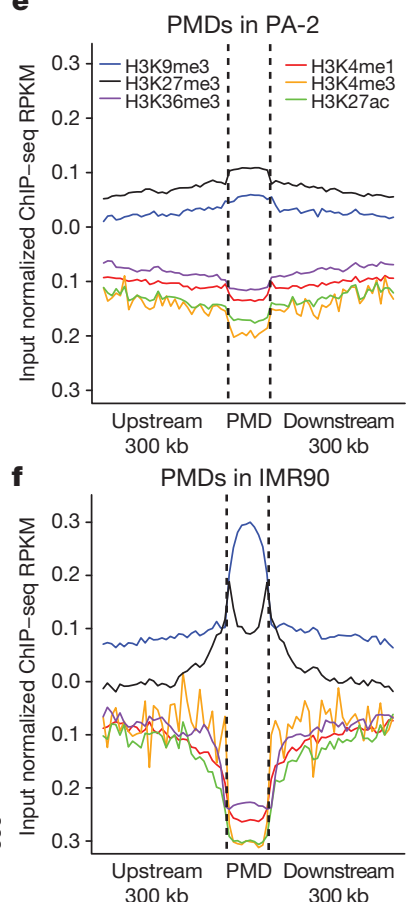

Figure $2 \mid$ DNA methylation and its relationship with gene expression. a, The mean Spearman correlation coefficient at various distances between the methylation level of autosomal DMRs and the expression of the nearest gene. These correlations are shown for DMRs: overlapping genes (gene body), overlapping enhancers, overlapping promoters or CpG islands (CGIs) or CGI shores, not overlapping genes (intergenic) and all remaining DMRs (undefined). TSS, transcription start site. b. Heatmap showing the tissuespecific methylation preference of each motif. The tissues are coloured according to Fig. 1c, and the ordering is listed at the bottom of the figure.
The bar plot on the right shows the number of times the motif was present in the 20 motif models. c, The number of base pairs covered by PMDs in all samples. d, The distribution of expression inside and outside of PA-2 PMDs across various samples. Notches indicate a confidence interval estimated from 1,000 bootstrap samples. Each PMD boxplot consists of 3,627 genes, and each non-PMD boxplot consists of 22,907 genes. FPKM, fragments per kilobase of transcript per million mapped reads. e, $\mathbf{f}$, Histone modification profiles in and around PMDs in PA-2 (e) and IMR90 (f). systematic expression difference between samples with and without PMDs (Extended Data Fig. 7a-d).

Previous studies have highlighted the existence of methylation outside of the CG context $(\mathrm{mCH})$ in human embryonic stem cells ${ }^{4}$, brain $^{1,20}$ and at the promoter of the PGC-1 $\alpha$ gene $(P P A R G C 1 A)$ in skeletal muscle ${ }^{27}$. We found evidence for appreciable amounts of $\mathrm{mCH}$ in many of these tissues (Fig. 3a and Extended Data Fig. 8a). A 5-bp motif split the samples into two groups, one with $\mathrm{mCH}$ enriched in a TNCAC motif and another with $\mathrm{mCH}$ enriched in an NNCAN motif (where $\mathrm{N}$ is any base) (Supplementary Methods). The TNCAC motif is highly similar to the one previously identified in purified glia (GLA) and neurons (NRN) (TACAC). These motifs differ from those found in $\mathrm{H} 1$ embryonic stem cells (H1) and induced pluripotent stem cells (TACAG) $)^{4,26}$ (Fig. 3b-d). We quantified the extent of $\mathrm{mCH}$ across these samples by plotting the distribution of methylation levels at $\mathrm{mCH}$ sites in the 25 samples with a TNCAC motif, which revealed a methylation level similar to that of GLA, NRN and H1 (Extended Data Fig. 8b) ${ }^{4,20}$. Most of the tissue types were consistently enriched for the TNCAC or NNCAN motif, but several (oesophagus, lung, pancreas and spleen) had replicates that disagreed, suggesting that $\mathrm{mCH}$ is not homogenously distributed across these tissues.

To examine the potential functional effect of $\mathrm{mCH}$ in adult tissues, we plotted the distribution of expression levels for various quantiles of gene body $\mathrm{mCH}$ as it was previously reported to be positively correlated with expression in $\mathrm{H} 1$ (ref. 4) and negatively correlated with expression in neurons ${ }^{20}$. This analysis revealed a negative correlation between expression and $\mathrm{mCH}$ (Extended Data Fig. $8 \mathrm{c}$ and Supplementary Methods). Next, we combined our replicates and clus- tered genes by the patterns of CAS methylation (in which S is a G or C) in and around their gene body (Fig. 3e and Supplementary Methods). To characterize the genes assigned to each cluster, we performed DAVID functional annotation clustering (Supplementary Table 9 and Supplementary Methods), which revealed several different classes. Clusters 1, 2, 16 and 19 contained genes highly enriched for terms involved in basic cellular processes and had an active methylation state (that is, hypermethylation in embryonic samples and hypomethylation in tissue and brain samples) across all samples. Clusters 5 and 6 were dominated by terms related to neuronal function and genes in this class were differentially methylated between neurons and glia and have inactive methylation states in other samples (that is, hypomethylation in embryonic samples and hypermethylation in tissue and brain samples). Cluster 12 was enriched for heart- and muscle-related terms and its genes had an active methylation state in the three heart tissues as well as a weakly active methylation state in psoas but appeared inactive in other samples. Lastly, cluster 14 possessed an active methylation state in brain and tissue samples but was inactive in embryonic samples. Despite being inactive in the $\mathrm{H} 1$ samples, this class of genes was highly enriched for terms related to development.

To define the transition of $\mathrm{mCH}$ motifs over development better, we examined the ratio of the methylation level of CAC and CAG (mCAC and $\mathrm{mCAG}$ ) sites in a variety of differentiated (tissues, NRN and GLA), embryonic (H1), and embryonic-derived (neural progenitor cells (NPC), mesendoderm (MES), trophoblast-like (TRO), mesenchymal stem cells (MSC) $)^{28}$ cell samples (Fig. 3f). With the exception of brain cells, $\mathrm{mCH}$ levels drop during differentiation, and the mCAC/mCAG ratios revealed a shift in motif usage across developmental time (Fig. 3f); although, mCAC and $\mathrm{mCAG}$ within the same gene remain 


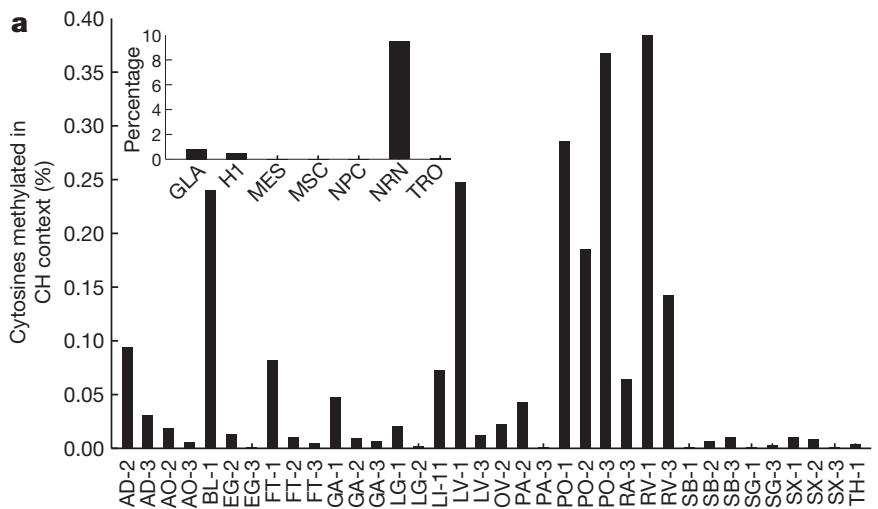

b

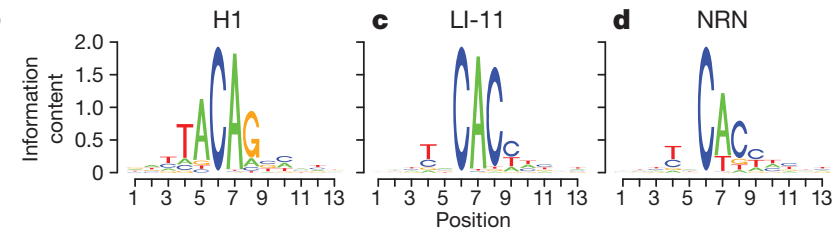

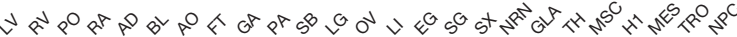

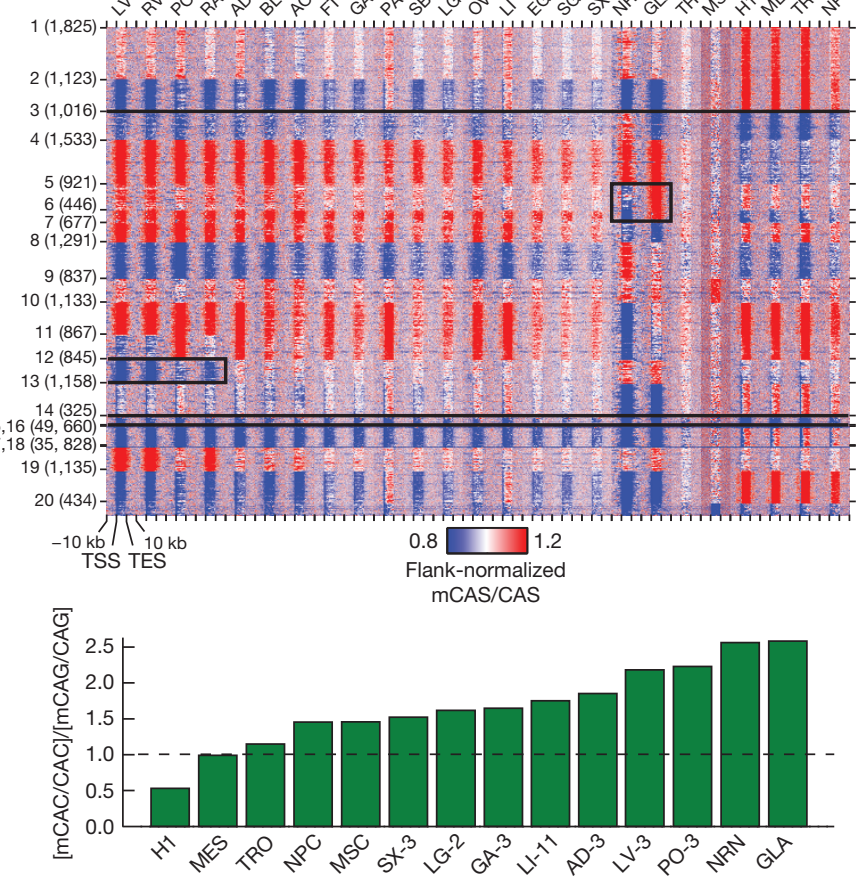

Figure $3 \mid \mathbf{m C H}$ is prevalent in human tissues. a, The fraction of methylated cytosines in the $\mathrm{CH}$ context by sample. $\mathbf{b}-\mathbf{d}$, Representative $\mathrm{mCH}$ motifs from embryonic $(\mathrm{H} 1 ; \mathbf{b})$, tissue (LI- $11 ; \mathbf{c})$ and brain $(\mathrm{NRN} ; \mathbf{d})$ samples. The height of each letter represents its information content. e, Heatmap of genic mCAS patterns normalized to the flanking region. Each gene was assigned to 1 of 20 clusters, which is indicated by the number and tick marks on the $y$ axis. The tick marks on the $x$ axis indicate the upstream, transcription start, transcription end, and downstream segments of each gene. The boxes around various patterns highlight regions referenced in the main text. TES, transcription end site. f, Bar plot of the ratio of the genome-wide mCAC to $\mathrm{mCAG}$ in various samples.

tightly correlated in both early embryonic and differentiated tissues (Extended Data Fig. 8d, e).

Methylation has previously been shown to be predictive of genes escaping $\mathrm{X}$-chromosome inactivation in neurons ${ }^{20}$. We investigated this phenomenon in these samples by comparing the promoter mCG and gene body $\mathrm{mCH}$ of genes that had previously been identified to escape X-chromosome inactivation ${ }^{29}$ in 11 tissues with $\mathrm{mCH}$ (Fig. 4a). Female-specific promoter mCG hypomethylation and gene body $\mathrm{mCH}$ hypermethylation were present at escapee genes at a similar
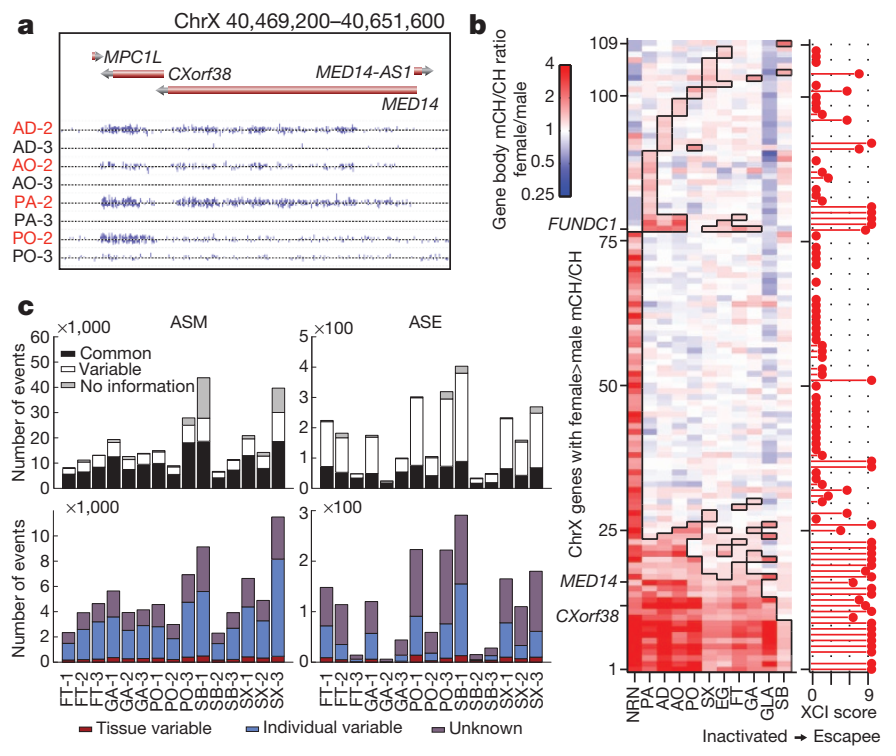

Figure 4 | Allele-specific methylation and expression. a, Browser screenshot of the increase in female $\mathrm{mCH}$ for a gene known to escape $\mathrm{X}$-chromosome inactivation (MED14). Sample names are coloured by gender (male, black; female, red). MED14-AS1 is also known as MED14OS. b, Ratio of $\mathrm{mCH}$ level in female versus male samples across genes with a significant difference in at least one sample. Cells boxed in black denote samples with a statistically significant difference between females and males. The XCI score for each gene is from ref. 29 and indicates the degree of escaping $\mathrm{X}$-chromosome inactivation. c, The number of ASM and ASE sites across the triplicated tissues. The top row depicts ASM events (left) and ASE events (right) that are allele-specific in all tissues (black), are variable across tissues (white), or do not possess enough data to tell (grey). The bottom row depicts the distribution of variable sites from the top row that vary by individual (blue), tissue (red) or neither (purple).

level as in neurons ${ }^{20}$ (Extended Data Fig. 9a). Using these tissue methylomes, gene body $\mathrm{mCH}$ was appreciably predictive of biallecially expressed genes (AUC 0.89; Extended Data Fig. $9 \mathrm{~b}$ and Supplementary Methods). To a lesser extent, we observed female-specific promoter $\mathrm{mCH}$ and gene body $\mathrm{mCG}$ hypermethylation at escapee genes (Extended Data Fig. 9a, c, d). Although female-specific promoter mCG hypomethylation, promoter $\mathrm{mCH}$ hypermethylation and gene body mCG hypermethylation are predictive of X-chromosome inactivation escapees, female-specific gene body $\mathrm{mCH}$ hypermethylation is the most predictive feature of $\mathrm{X}$-chromosome inactivation escapees (Extended Data Fig. 9a, b-e). We detected female-specific $\mathrm{mCH}$ hypermethylation in 109 out of $612 \mathrm{X}$-linked genes, including 9 genes hypermethylated in all 11 tissues and 72 genes that were hypermethylated in only one tissue (Fig. 4b). Several genes such as FUNDC1 showed female-specific hypermethylation in several tissues but not in neurons, suggesting a tissue-dependent regulation of the escape from $\mathrm{X}$ inactivation.

Allele-specific methylation and expression (ASM and ASE, respectively) may also have a role in the regulation of autosomal genes. To examine these phenomena in human tissues, we combined the RNAseq and MethylC-seq data sets with phased genotypes for each individual in this study,15 (Extended Data Fig. 10a and Supplementary Methods). Using the triplicate tissue samples (fat (FT), gastric (GA), psoas (PO), small bowel (SB) and spleen (SX)), we identified 8,464-48,560 ASM events in the CG context and 48-403 ASE genes across these tissues (Supplementary Tables 10, 11 and Supplementary Methods). We next looked for ASM events that varied across individuals within a tissue-type (tissue variable) and those that varied across a tissue-type within an individual (individual variable). Of the ASM events that varied, $4.1-7.5 \%$ and $54.5-70.0 \%$ were tissue- and individual-variable, respectively; whereas, of the ASE events that varied, $0.0-20.0 \%$ were individual-variable and $13.3-48.8 \%$ were 
tissue-variable (Fig. 4c and Supplementary Methods). Of the ASE events, 38.4-87.4\% had an ASM event within $100 \mathrm{~kb}$, and of these sites, 76\% had an ASM and ASE event that was matched (that is, a DMR was hypomethylated on the same haplotype as the more highly expressed allele). Furthermore, we found that a larger fraction of ASE genes were observed near ASM events whether or not the events matched (Extended Data Fig. 10 b, c and Supplementary Methods). These results demonstrate a link between ASM and ASE in human tissues.

Here we have presented the deepest set of base resolution maps of $\mathrm{mCG}$ and $\mathrm{mCH}$ so far along with chromatin modification states, haplotype-resolved genome sequences and transcriptional profiles for a large set of human tissues. These data sets allowed us to identify cis-regulatory elements accurately. Furthermore, they revealed the existence of $\mathrm{mCH}$ genome-wide in a subpopulation of cells from differentiated human tissues, which seems to be repressive. Our analysis of genic $\mathrm{mCH}$ across human tissues indicates a tissue-specific distribution that is distinct from those genes that were previously identified in embryonic stem cells and the brain. These genes are enriched for a variety of functions, most surprisingly those involved in development. These analyses raise the intriguing possibility that $\mathrm{mCH}$ is used in adult stem cells ${ }^{30}$ and could help to repress these genes as the cells transition into their differentiated role.

Received 25 November 2013; accepted 13 April 2015.

Published online 1 June 2015.

1. Varley, K. E. et al. Dynamic DNA methylation across diverse human cell lines and tissues. Genome Res. 23, 555-567 (2013).

2. Ziller, M. J. et al. Charting a dynamic DNA methylation landscape of the human genome. Nature 500, 477-481 (2013).

3. Selvaraj, S., Dixon, J. R., Bansal, V. \& Ren, B. Whole-genome haplotype reconstruction using proximity-ligation and shotgun sequencing. Nature Biotechnol. 31, 1111-1118 (2013).

4. Lister, R. et al. Human DNA methylomes at base resolution show widespread epigenomic differences. Nature 462, 315-322 (2009).

5. Irizarry, R. A. et al. The human colon cancer methylome shows similar hypo- and hypermethylation at conserved tissue-specific CpG island shores. Nature Genet. 41, 178-186 (2009).

6. Hon, G. C. et al. Epigenetic memory at embryonic enhancers identified in DNA methylation maps from adult mouse tissues. Nature Genet. 45, 1198-1206 (2013).

7. Hnisz, D. et al. Super-enhancers in the control of cell identity and disease. Cell 155, 934-947 (2013).

8. Yuen, S. L., Ogut, O. \& Brozovich, F. V. Nonmuscle myosin is regulated during smooth muscle contraction. Am. J. Physiol. Heart Circ. Physiol. 297, H191-H199 (2009).

9. Schultz, M. D., Schmitz, R. J. \& Ecker, J. R. 'Leveling' the playing field for analyses of single-base resolution DNA methylomes. Trends Genet. 28, 583-585 (2012).

10. McLean, C. Y. et al. GREAT improves functional interpretation of cis-regulatory regions. Nature Biotechnol. 28, 495-501 (2010).

11. Hovestadt, V. et al. Decoding the regulatory landscape of medulloblastoma using DNA methylation sequencing. Nature 510, 537-541 (2014).

12. Maunakea, A. K. et al. Conserved role of intragenic DNA methylation in regulating alternative promoters. Nature 466, 253-257 (2010).

13. Doi, A. et al. Differential methylation of tissue- and cancer-specific $\mathrm{CpG}$ island shores distinguishes human induced pluripotent stem cells, embryonic stem cells and fibroblasts. Nature Genet. 41, 1350-1353 (2009).

14. Deaton, A. M. et al. Cell type-specific DNA methylation at intragenic $\mathrm{CpG}$ islands in the immune system. Genome Res. 21, 1074-1086 (2011).

15. Leung, D. et al. Integrative analysis of haplotype-resolved epigenomes across human tissues. Nature 518, 350-354 (2015).

16. Parviz, F. et al. Hepatocyte nuclear factor 4 alpha controls the development of a hepatic epithelium and liver morphogenesis. Nature Genet. 34, 292-296 (2003)

17. Maurano, M. T. et al. Systematic localization of common disease-associated variation in regulatory DNA. Science 337, 1190-1195 (2012).

18. Gutierrez-Arcelus, M. et al. Passive and active DNA methylation and the interplay with genetic variation in gene regulation. Elife 2, e00523 (2013).
19. Liu, Y. et al. Epigenome-wide association data implicate DNA methylation as an intermediary of genetic risk in rheumatoid arthritis. Nature Biotechnol. 31, 142-147 (2013).

20. Lister, R. et al. Global epigenomic reconfiguration during mammalian brain development. Science 341, 6146 (2013).

21. Whitaker, J. W., Chen, Z. \& Wang, W. Predicting the human epigenome from DNA motifs. Nature Methods 12, 265-272 (2015).

22. Stankunas, K. et al. Pbx/Meis deficiencies demonstrate multigenetic origins of congenital heart disease. Circ. Res. 103, 702-709 (2008).

23. Hon, G. C. et al. Global DNA hypomethylation coupled to repressive chromatin domain formation and gene silencing in breast cancer. Genome Res. 22, 246-258 (2012).

24. Berman, B. P. et al. Regions of focal DNA hypermethylation and long-range hypomethylation in colorectal cancer coincide with nuclear lamina-associated domains. Nature Genet. 44, 40-46 (2011).

25. Schroeder, D. I. et al. The human placenta methylome. Proc. Natl Acad. Sci. USA $110,6037-6042$ (2013).

26. Lister, R. etal. Hotspots of aberrant epigenomic reprogramming in human induced pluripotent stem cells. Nature 471, 68-73 (2011).

27. Barrès, R. et al. Non-CpG methylation of the PGC-1 $\alpha$ promoter through DNMT3B controls mitochondrial density. Cell Metab. 10, 189-198 (2009).

28. Xie, W. et al. Epigenomic analysis of multilineage differentiation of human embryonic stem cells. Cell 153, 1134-1148 (2013).

29. Carrel, L. \& Willard, H. F. X-inactivation profile reveals extensive variability in X-linked gene expression in females. Nature 434, 400-404 (2005).

30. Wagers, A. J. \& Weissman, I. L. Plasticity of adult stem cells. Cell 116, 639-648 (2004).

Supplementary Information is available in the online version of the paper.

Acknowledgements We thank R. J. Schmitz for critical reading of the manuscript. This work is supported by the National Institutes of Health $(\mathrm{NIH})$ Epigenome Roadmap Project (U01 ES017166). E.A.M. was supported by National Institute of Neurological Diseases and Stroke grant (R0ONS080911). J.R.E. was supported by the Gordon and Betty Moore Foundation (GMBF3034) and the Mary K. Chapman Foundation. T.J.S. and J.R.E. are investigators of the Howard Hughes Medical Institute. S.L. was supported by NIH fellowship grants F32HL110473 and K99HL119617. The authors acknowledge the Texas Advanced Computing Center (TACC) at The University of Texas at Austin for providing HPC resources that have contributed to the research results reported within this paper. The authors would also like to thank Mid-America Transplant Services, St Louis, for their support of this research effort.

Author Contributions B.R., T.J.S., W.W. and J.R.E. designed and supervised research. S.L. and Y.L. collected tissues. J.R.N. and M.A.U. conducted MethylC-seq, RNA-seq and genome sequencing experiments. D.L. conducted ChIP-seq experiments. N.R. performed ChIP-seq data analysis. M.D.S., Y.H., M.H. and H.C. performed sequencing data processing. J.W.W. performed motif prediction and mutation analysis. M.D.S. designed and implemented the methylation processing and analysis module. M.D.S., Y.H., J.W.W., M.H. and E.A.M. performed statistical and bioinformatic analyses. M.D.S., Y.H., J.W.W. and J.R.E. prepared the manuscript.

Author Information The sequencing data sets generated for this study as well as those for the IMR9O, $\mathrm{H} 1$ and $\mathrm{H} 1$ derived samples can be found at the Gene Expression Omnibus (GEO) under the accession number GSE16256. The sequencing data sets for the fetal tissues used in this study can be found at GEO under the accession number GSE18927. The sequencing data sets for the placental tissue used in this study can be found at GEO under the accession number GSE39777. The sequencing data sets for the neuronal and glial samples can be found at GEO under the accession number GSE47966 (NRN GSM1173776; GLA GSM1173777). The human tissue sequencing data generated for this study can be found at Sequence Read Archive (SRA) under the project number SRP000941. Analysed data sets can be obtained from http:// neomorph.salk.edu/human_tissue_methylomes.html. Reprints and permissions information is available at www.nature.com/reprints. The authors declare no competing financial interests. Readers are welcome to comment on the online version of the paper. Correspondence and requests for materials should be addressed to J.R.E. (ecker@salk.edu).

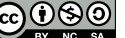

This work is licensed under a Creative Commons AttributionNonCommercial-ShareAlike 3.0 Unported licence. The images or other third party material in this article are included in the article's Creative Commons licence, unless indicated otherwise in the credit line; if the material is not included under the Creative Commons licence, users will need to obtain permission from the licence holde to reproduce the material. To view a copy of this licence, visit http://creativecommons. org/licenses/by-nc-sa/3.0 


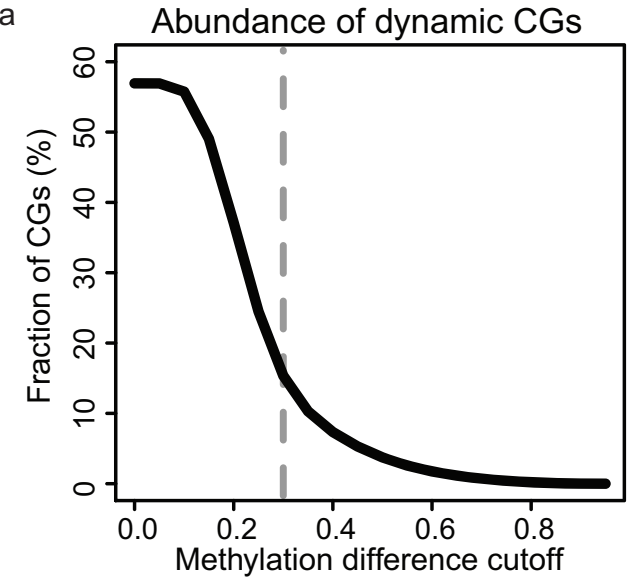

C

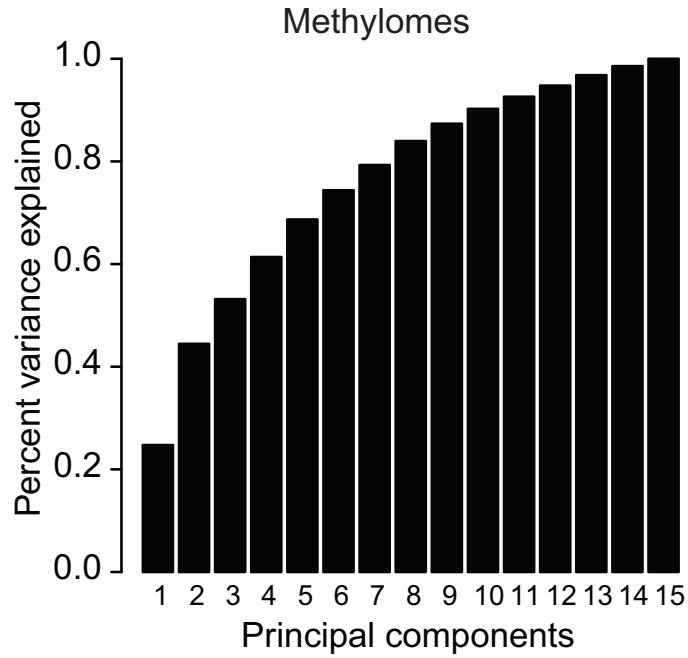

b

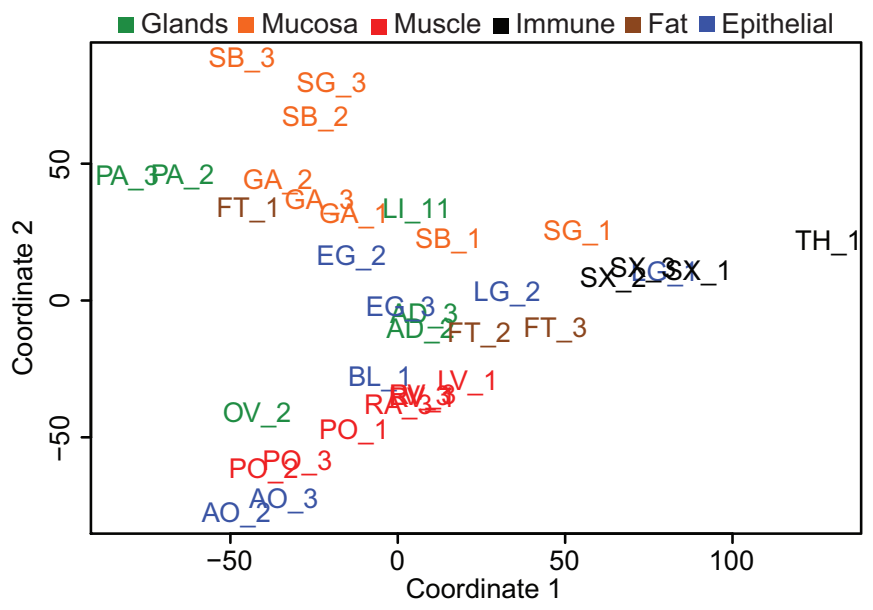

d

Transcriptomes

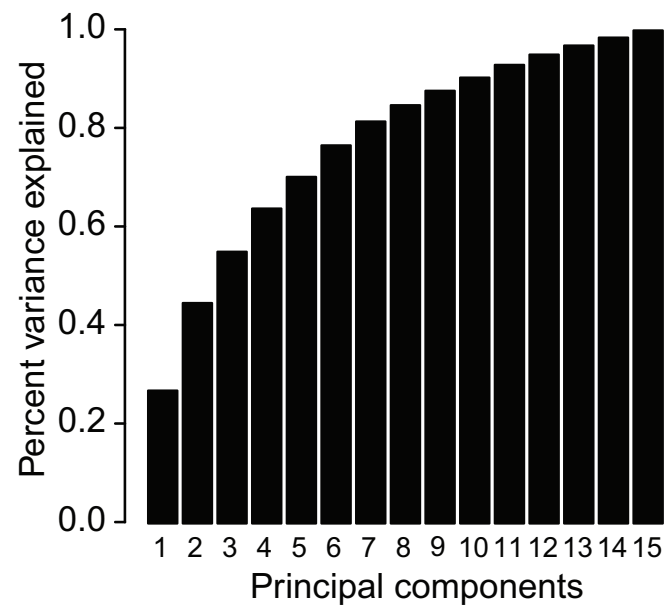

e

GO Biological Process

$-\log 10$ (Binomial $p$ value)

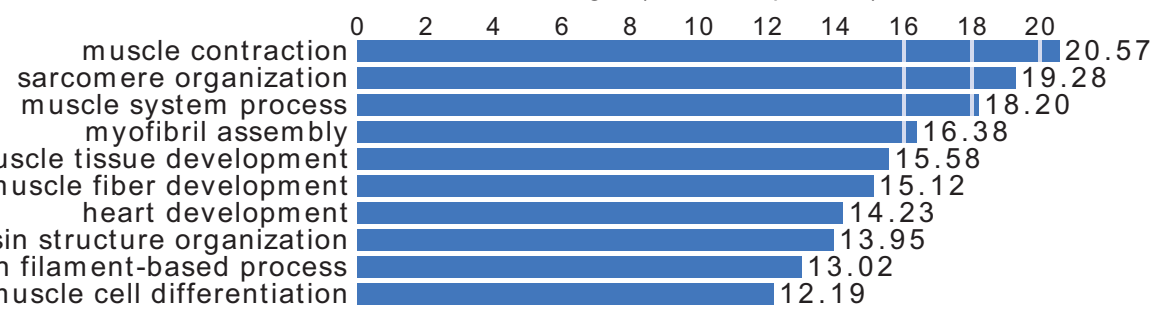

Mouse Phenotype

$-\log 10$ (Binomial $p$ value) cardiac muscle fiber development actomyosin structure organization actin filament-based process
striated muscle cell differentiation f

$\begin{array}{lllllllllll}8 & 10 & 12 & 14 & 16 & 18 & 20 & 22 & 24 & 26 & 28\end{array}$

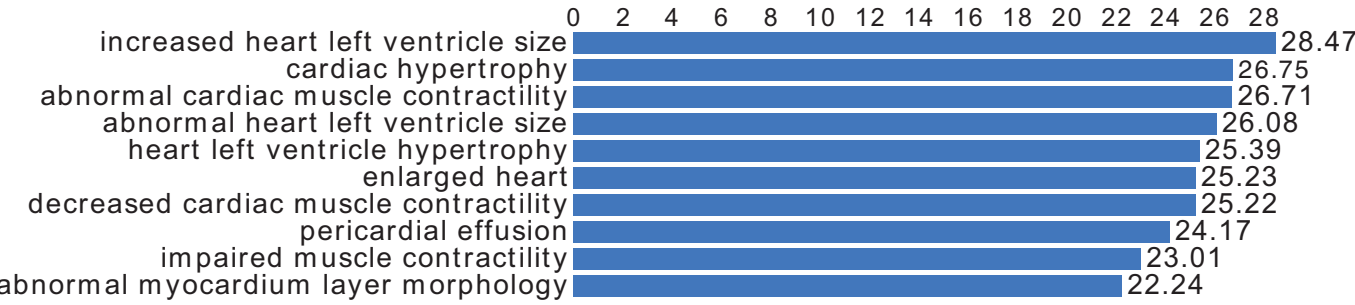

Extended Data Figure 1 Identification of DMRs and multidimensional scaling analysis. a, Line plot showing the fraction of differentially methylated CG sites (dynamic CGs) out of all CG sites under various methylation

difference cutoffs. The methylation difference of a CG site is defined in ref. 2. The grey line indicates the cutoff $(0.3)$ used to call differentially methylated sites. b, A plot of the first two principal components from the methylation level multidimensional scaling. Tissues are shaded by the organ group they belong to as in Fig. 1c, d. c, d, Bar charts of the cumulative amount of variance explained by the first $N$ principal components from the multidimensional scaling performed on the methylation levels of all DMRs (c) and the expression levels of all differentially expressed genes (d). e, A representative example of enriched Gene Ontology biological process terms based on the most hypomethylated DMRs from LV-1. f, A representative example of enriched mouse phenotype terms based on the most hypomethylated DMRs from LV-1. 


\section{RESEARCH LETTER}

a

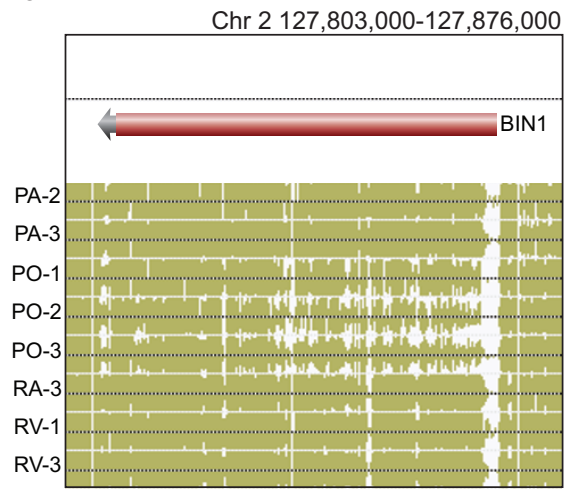

d uiDMR weak enhancer
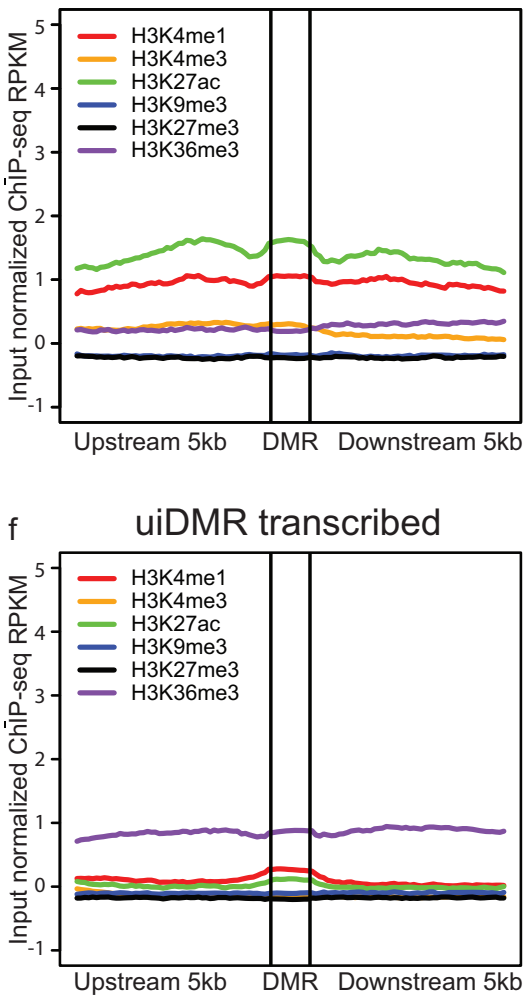

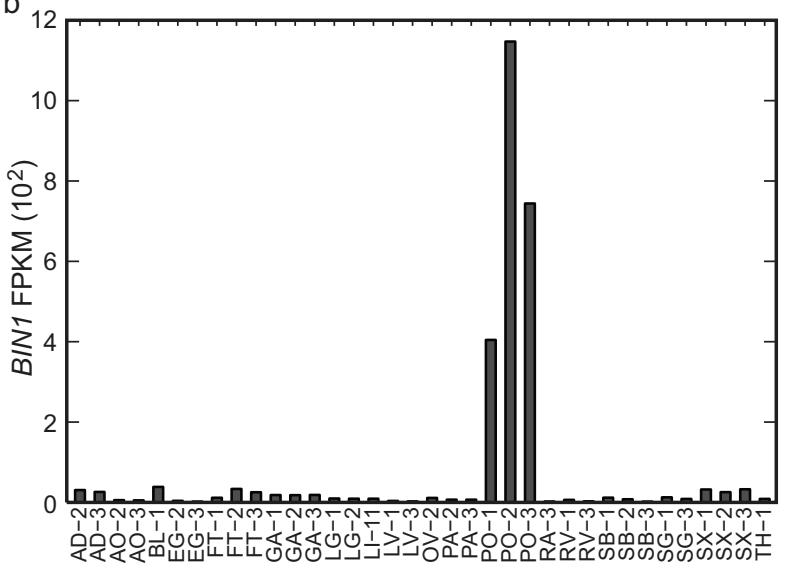

e uiDMR promoter-proximal

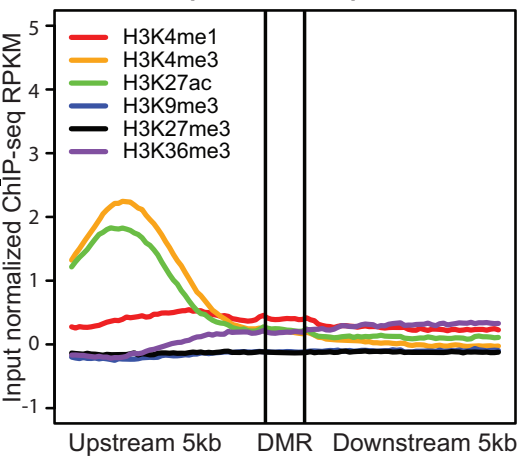

g

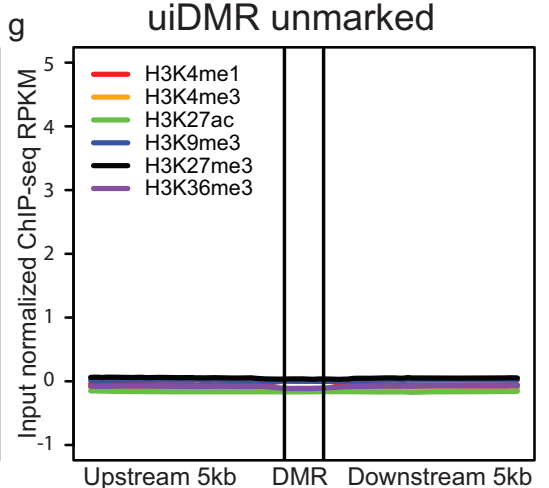

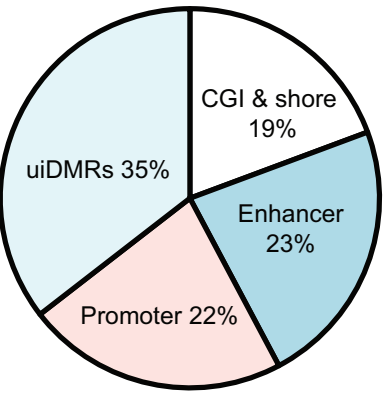

h uiDMR poised enhancer

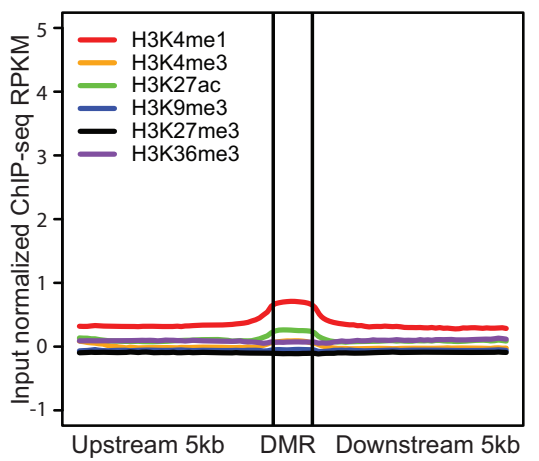

Extended Data Figure 2 $\mid$ DMRs and their correlation with transcription. a, A browser screenshot of an example DMR downstream of the transcription percentages of hypomethylated intragenic DMRs in each class of genomic features. $\mathbf{d}-\mathbf{h}$, Histone modification profiles of five categories of uiDMRs. 

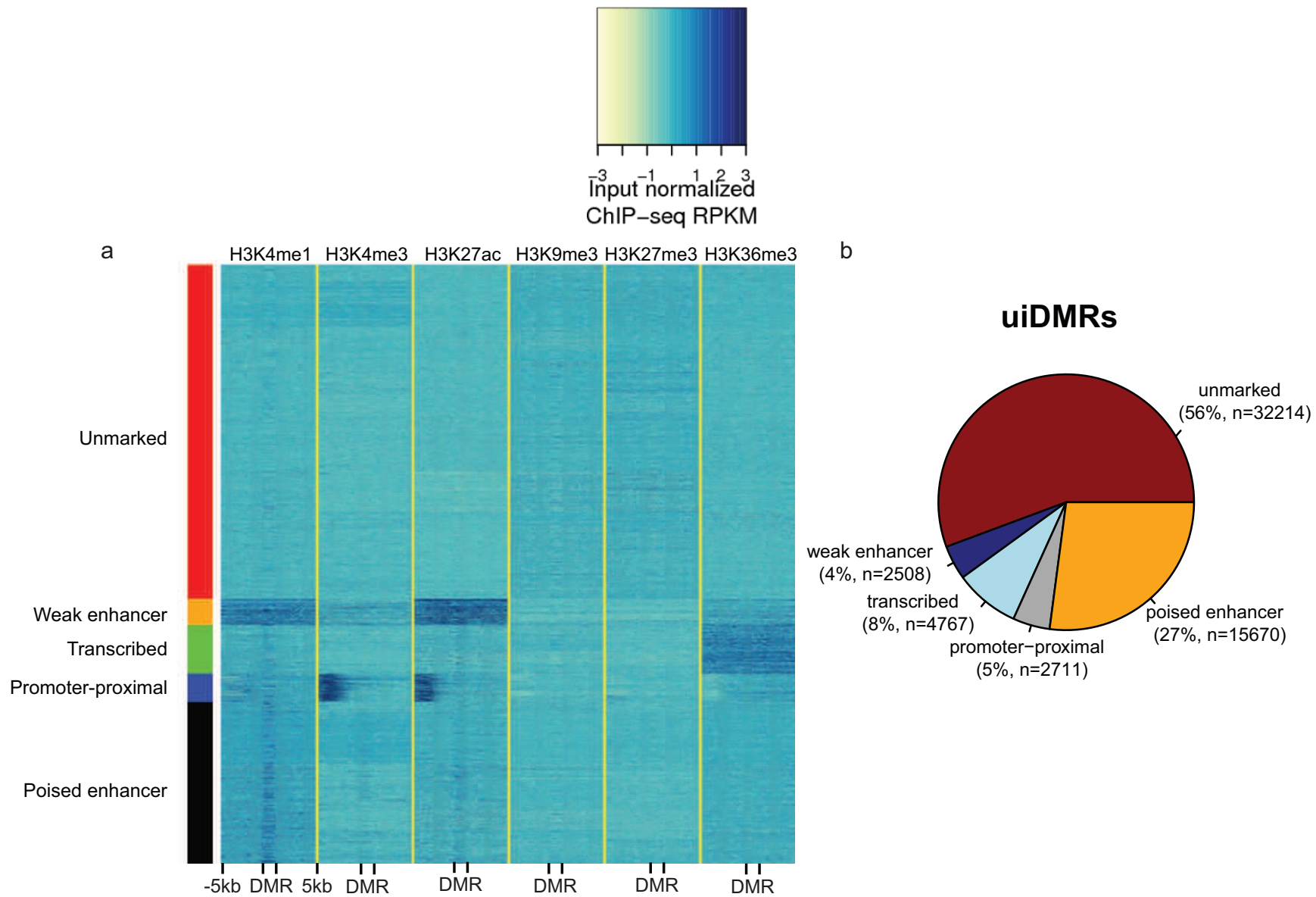

Extended Data Figure $3 \mid$ Classification of uiDMR histone profiles and uiDMR properties. a, Heatmap of the histone modification profiles for the five types of uiDMRs. The profiles were plotted for each mark across the DMR and the $5 \mathrm{~kb}$ upstream and downstream and the colours of each cell indicate the each profile assigned by $k$-means clustering (red, weak enhancer; orange, promoter-proximal; green, transcribed; blue, unmarked; black poised enhancer). b, A pie chart of the distribution of uiDMRs across the classes defined by $k$-means clustering. 
a

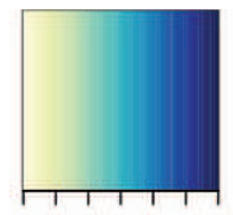

${ }^{3}{ }^{3}$ put ${ }^{-1}$ normalized

ChIP-seq RPKM

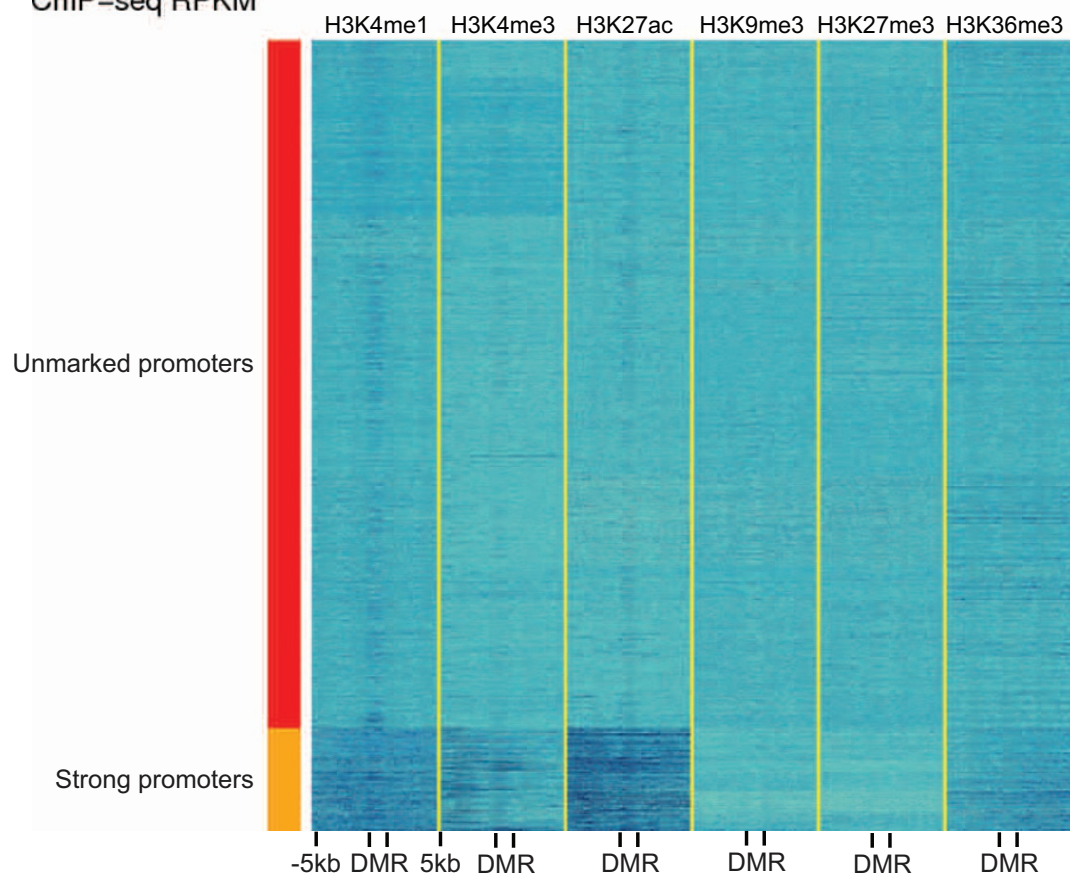

b

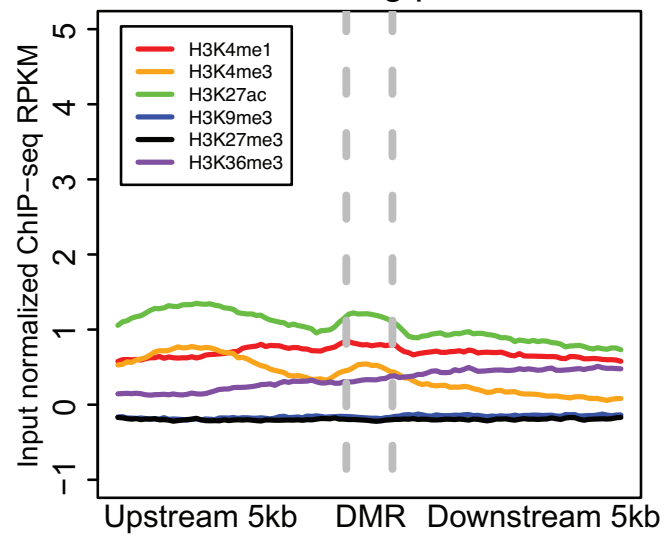

C

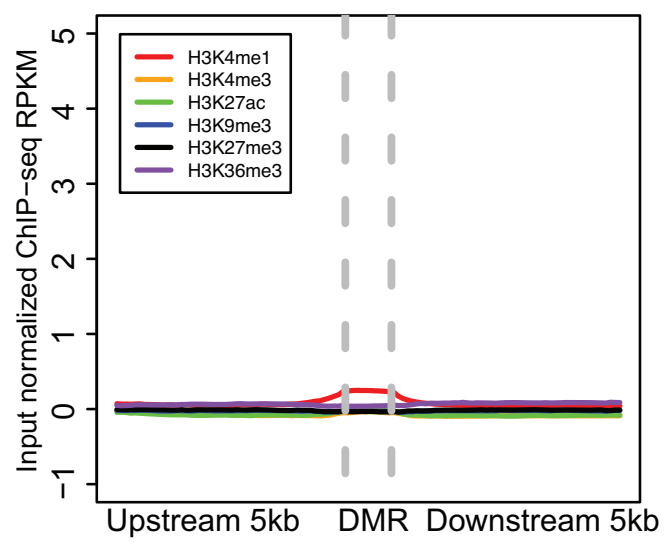

d

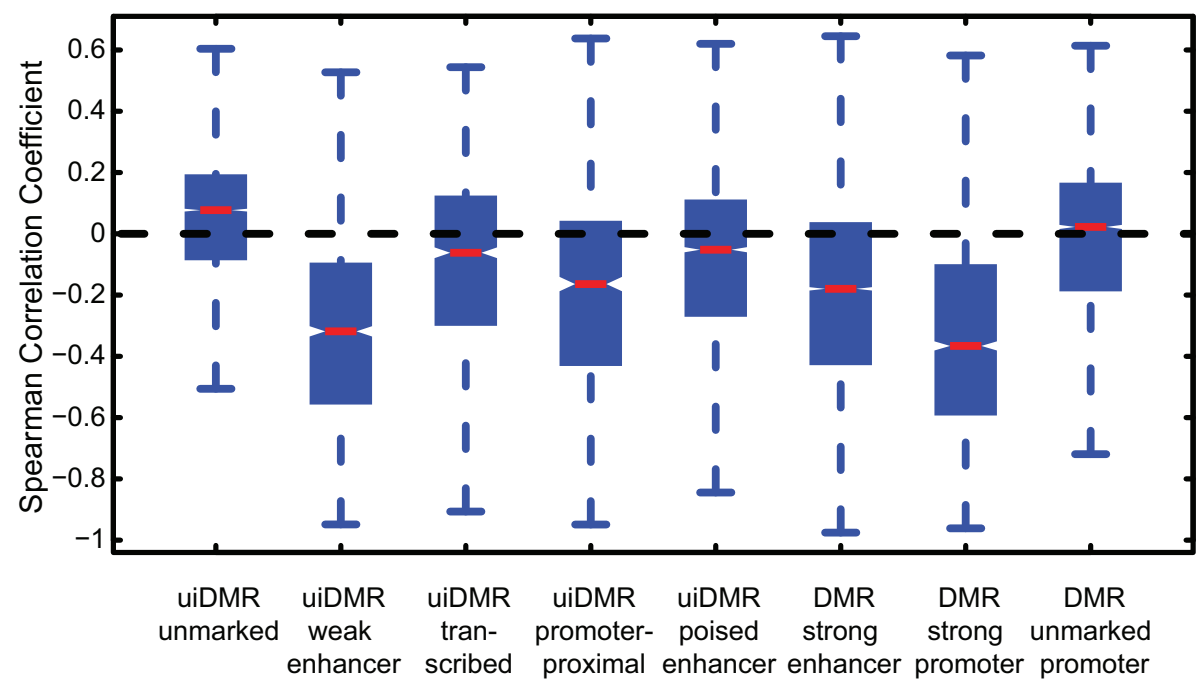

Extended Data Figure $4 \mid$ Classification of promoter histone profiles. a, A heatmap of the histone modification profiles across strong (rows labelled with red) and unmarked (rows labelled with orange) promoters. The profiles were plotted for each mark across the promoter and the 5-kb upstream and downstream, and the colours of each cell indicate the input normalized
ChIP-seq RPKM. b, c, The aggregate profiles for strong (b) and unmarked (c) promoters, respectively. d, The distribution of the Spearman correlation coefficients between the methylation level of different types of hypomethylated intragenic DMRs and the expression of the nearest gene. Notches indicate a confidence interval estimated from 1,000 bootstrap samples. 

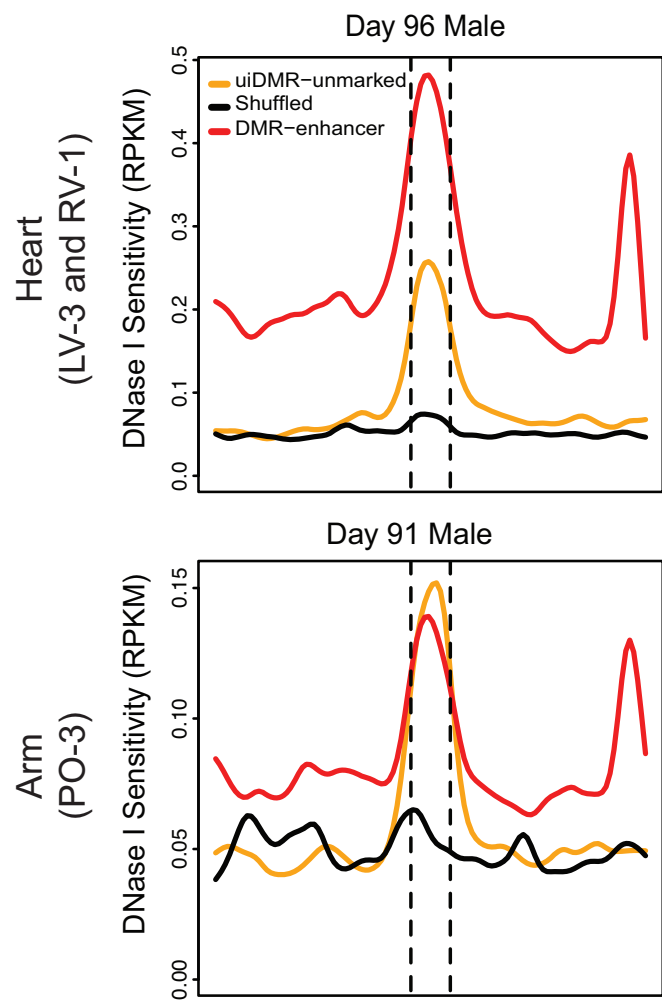

Day 91 Male
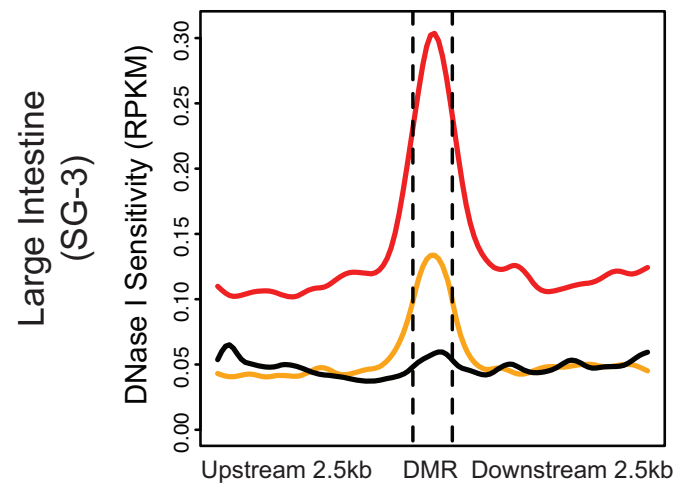

Day 105 Male

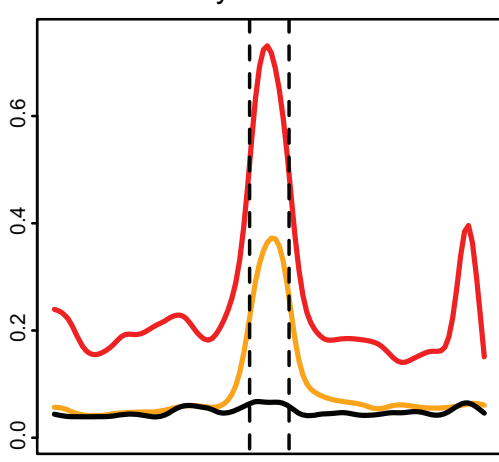

Day 115 Male

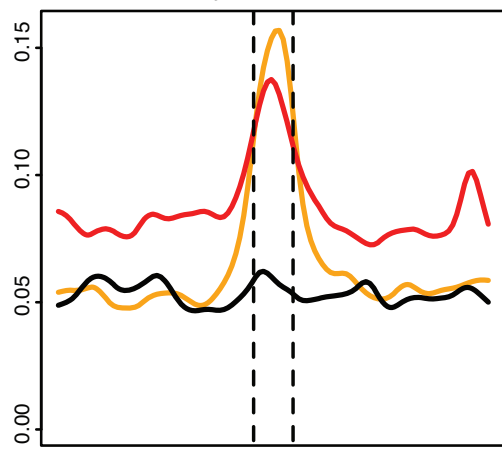

Day 105 Male

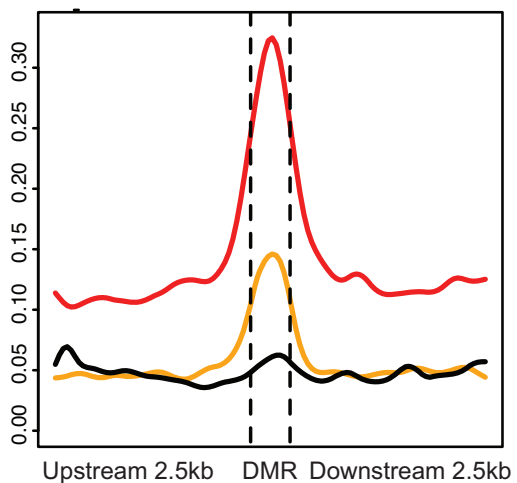

Day 110 Male

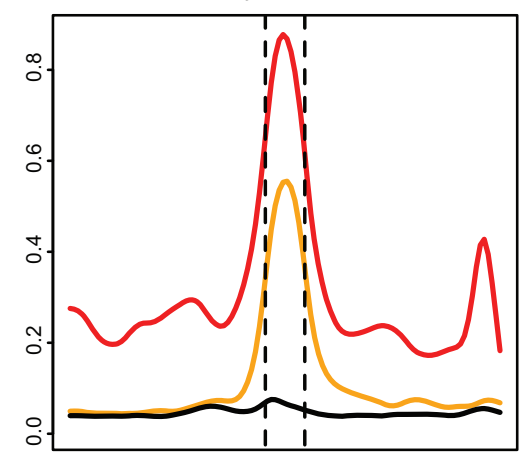

Day 120 Male

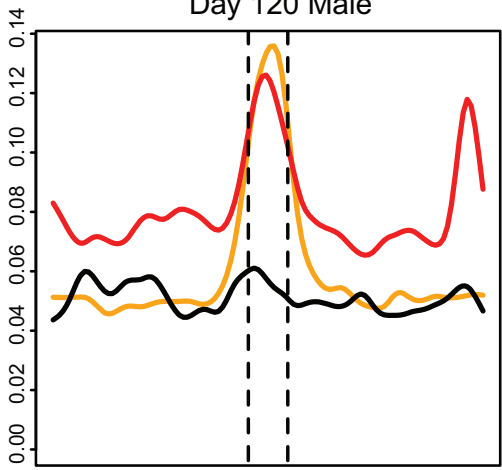

Day 115 Male

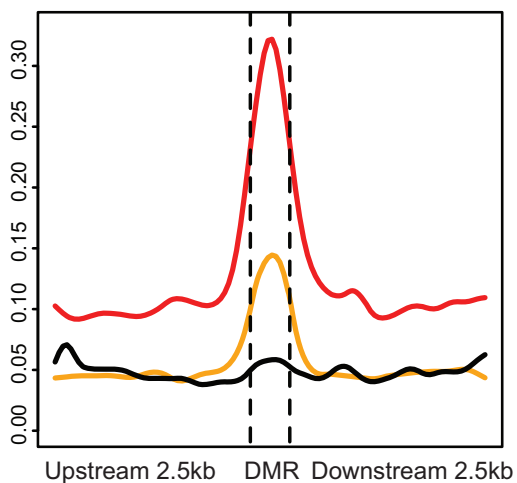

Extended Data Figure 5 | uiDMR fetal DNase I profiles. DNase I profiles of various fetal tissues corresponding to the tissues presented in this study. The samples are arranged column-wise by age, and row-wise by fetal tissue. The uiDMR-unmarked line represents the DNase I profile of uiDMRs without histone modifications. The DMR-enhancer line represents the DNase I profile of DMRs that overlapped an enhancer in a matched tissue in this study (indicated in the row label in parentheses). The shuffled line represents the DNase I profile of uiDMRs randomly shuffled across the genome. 


\section{RESEARCH LETTER}

a
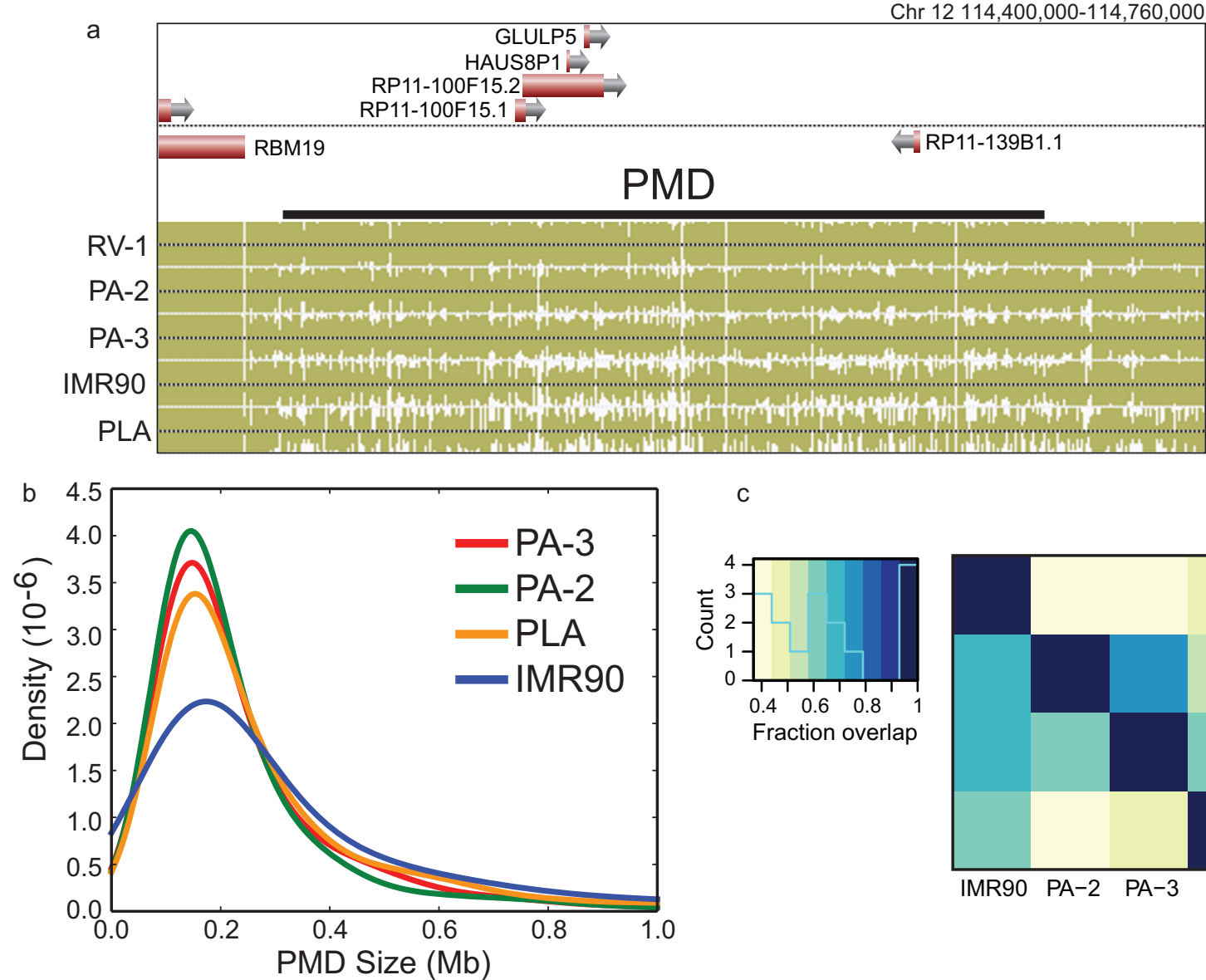

C

d
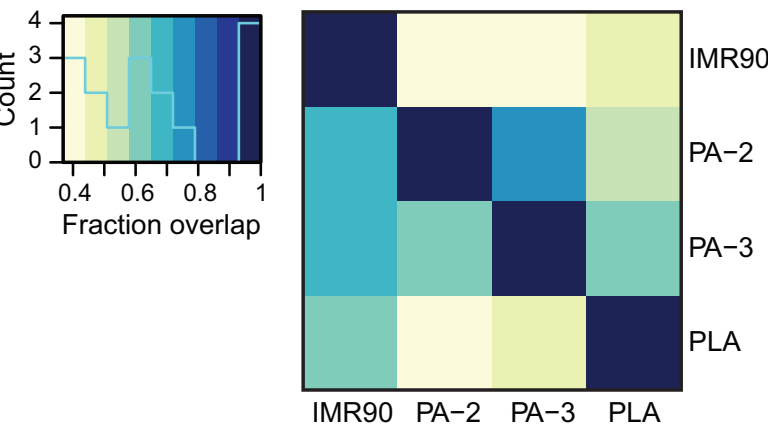

Shuffled PA-2 PMDs
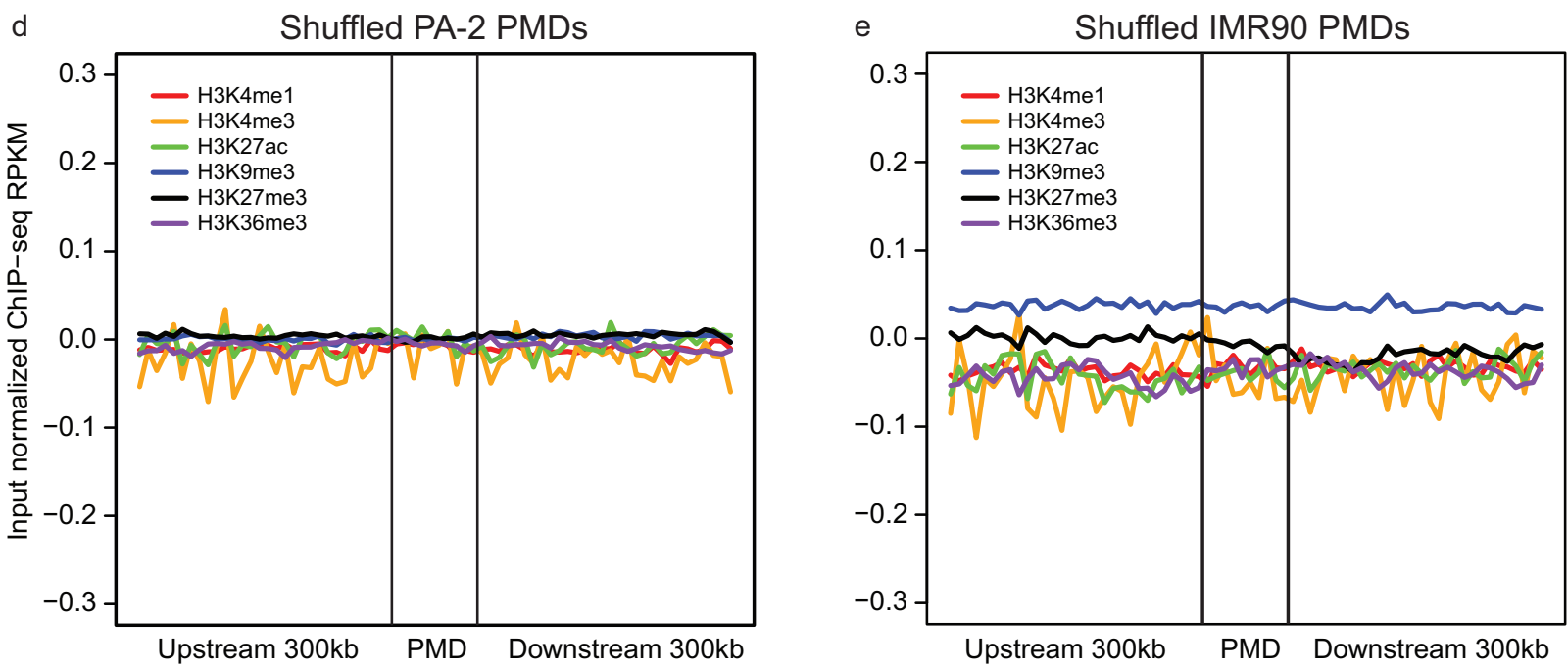

Extended Data Figure $6 \mid$ PMD features. a, A browser screenshot (see Fig. 1 for description) of an example PMD found in IMR90, PLA, PA-2 and PA-3. $\mathrm{RV}-1$ is included as a representative sample without PMDs. $\mathbf{b}$, The distribution of sizes of PMDs in various samples. c, A heatmap representation of the overlap

between various sets of PMDs. The denominator of the fraction of overlap is determined by the sample on the $y$ axis. d, e, ChIP-seq profiles of the PMD regions defined in PA-2 (c) and IMR90 (d) after shuffling. 
a
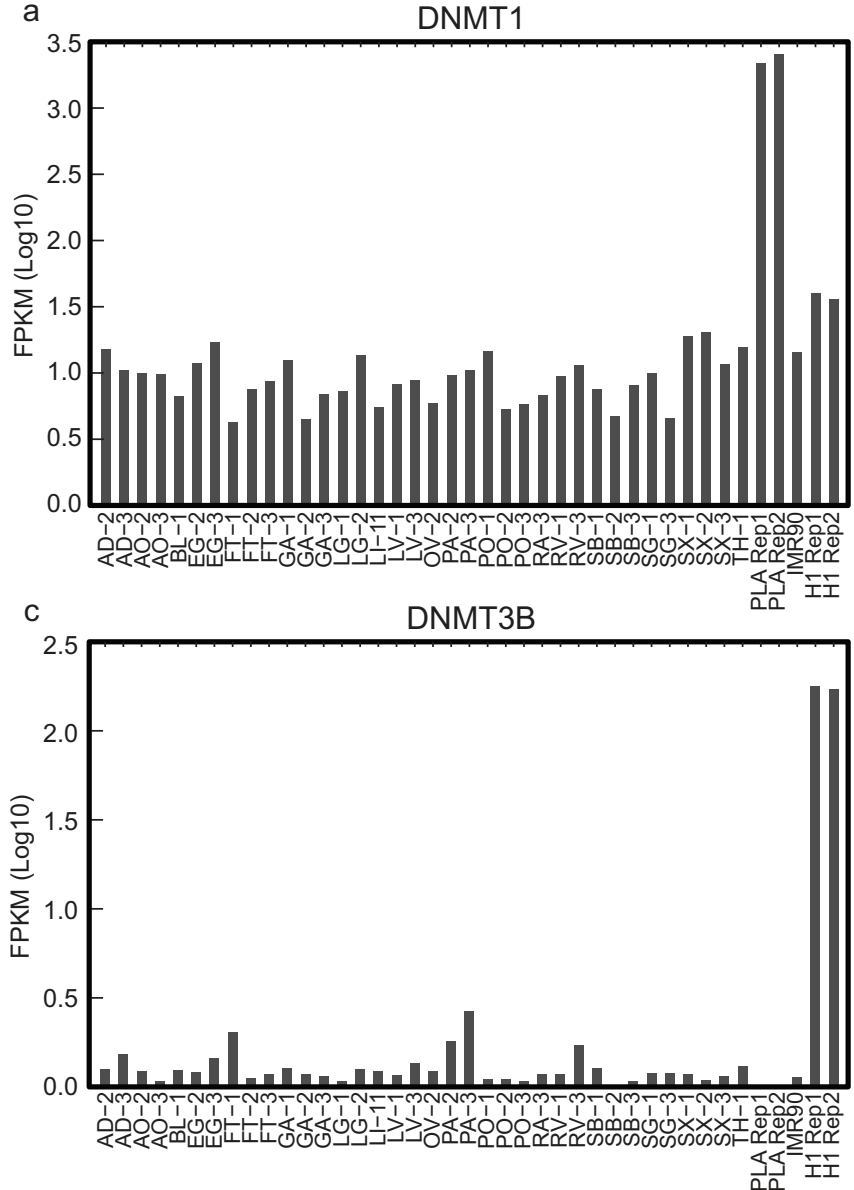

b

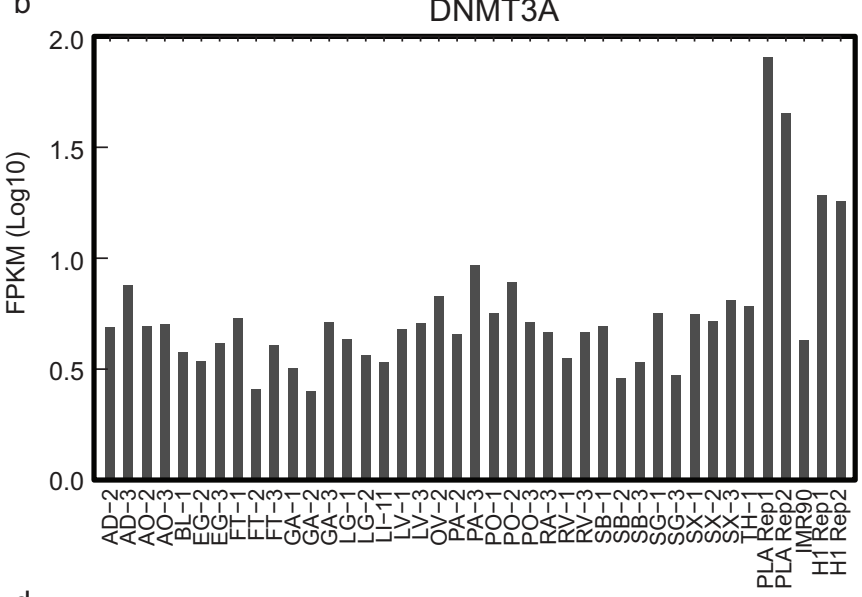

d

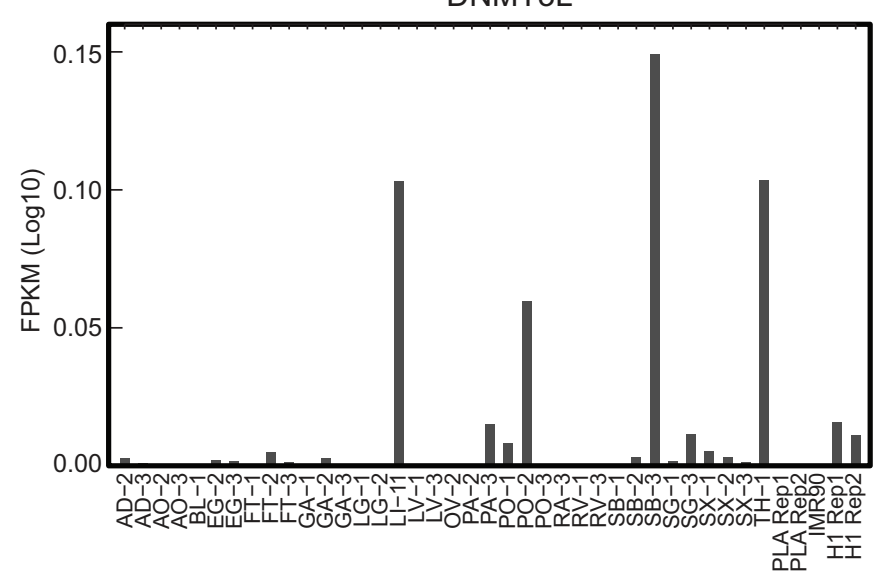

Extended Data Figure $7 \mid$ DNMT expression across tissues. a-d, Bar plots of the expression (measured in $\log _{10}$ FPKMs) of DNMT1 (a), DNMT3A (b), DNMT3B (c) and DNMT3L (d) across various samples. 

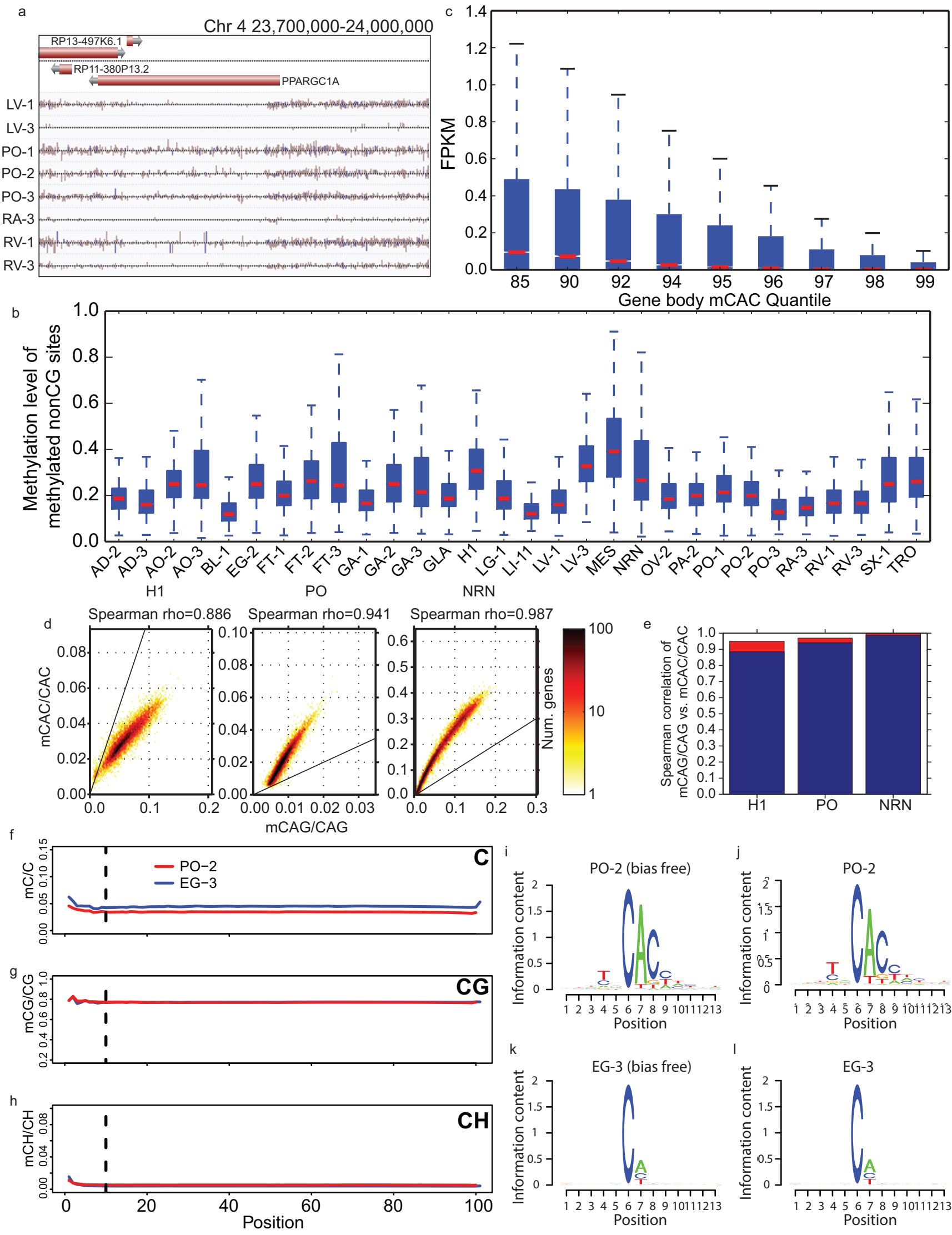
Extended Data Figure $8 \mid \mathrm{mCH}$ distribution and correlation. a, A browser screenshot (see Fig. 1 for description) of an example region with non-CG methylation $(\mathrm{mCH})$. Purple and pink ticks are methylated $\mathrm{CHG}$ and $\mathrm{CHH}$ sites, respectively $(\mathrm{H}=\mathrm{A}, \mathrm{C}$ or $\mathrm{T})$. Ticks on the forward strand are projected upward from the dotted line and ticks on the reverse strand are projected downward. b. The distribution of methylation levels at $\mathrm{mCH}$ sites across all samples with a discernible TNCAC motif. Only $\mathrm{mCH}$ sites with at least 10 reads and a significant amount of methylation were considered. c, Boxplots of the expression values across different quantiles of CAC gene body methylation (gene body mCAC). d, Scatterplot of $\mathrm{mCAG}$ versus $\mathrm{mCAC}$ inside gene bodies. e, Bar plot of the correlation of mCAG and mCAC inside gene bodies (blue) and the theoretical maximal correlation (red) if $\mathrm{mCAC}$ and $\mathrm{mCAG}$ are perfectly correlated. $\mathbf{f}-\mathbf{h}$, The methylation levels of $\mathrm{C}$ (top), CG (middle) and $\mathrm{CH}$ (bottom) across the read positions for PO-2 (red line) and EG-3 (blue line). Vertical lines indicate the position (tenth base from the beginning) where trimming was applied. $\mathbf{i}, \mathrm{mCH}$ motif from $\mathrm{PO}-2$ with the first 10 bases of each read trimmed. $\mathbf{j}$, $\mathrm{mCH}$ motif from $\mathrm{PO}-2$ without trimming. $\mathbf{k}$, $\mathrm{mCH}$ motif from EG-3 with the first 10 bases of each read trimmed. 1 , mCH motif from EG-3 without trimming. The height of each letter represents its information content (that is, prevalence). 


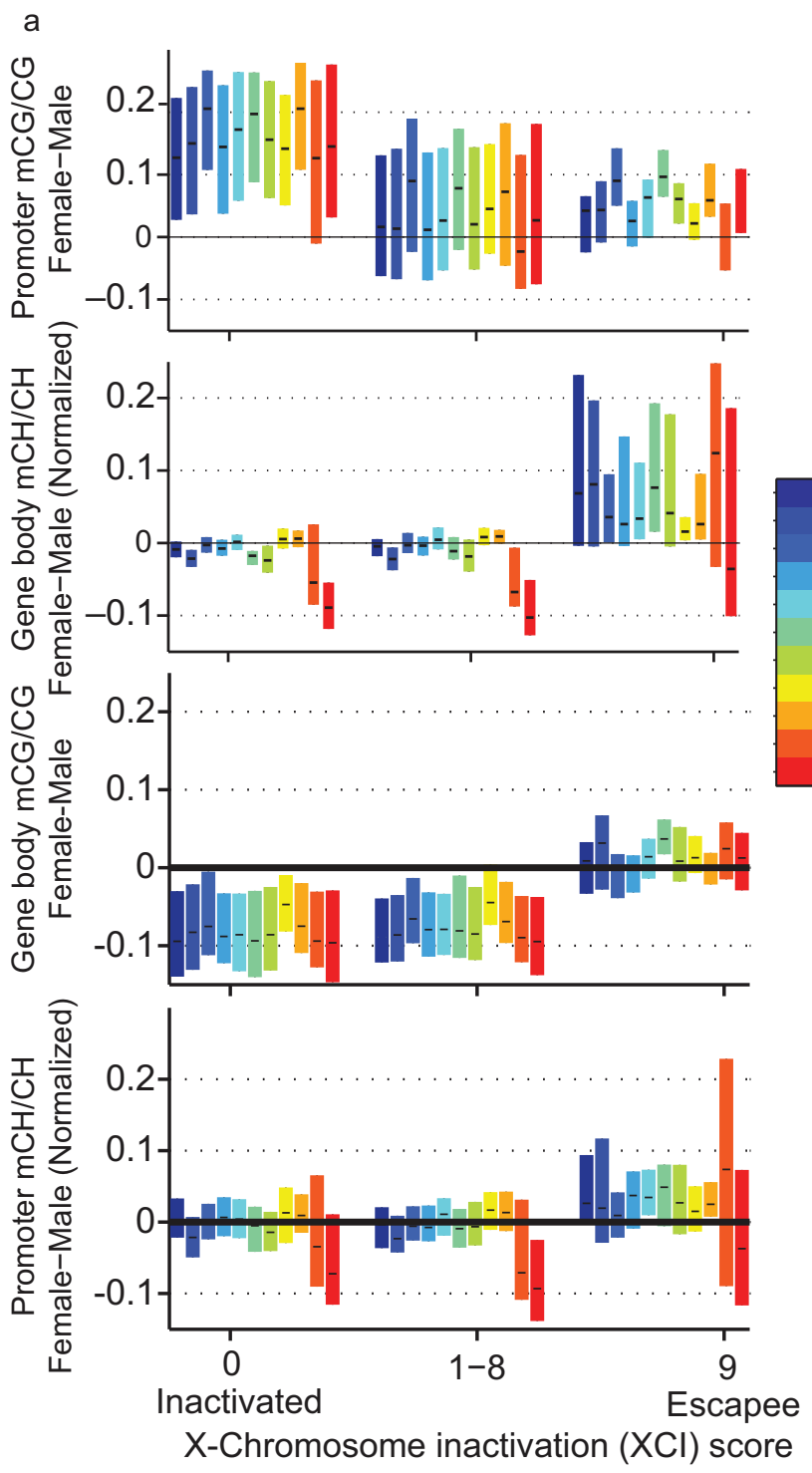

b
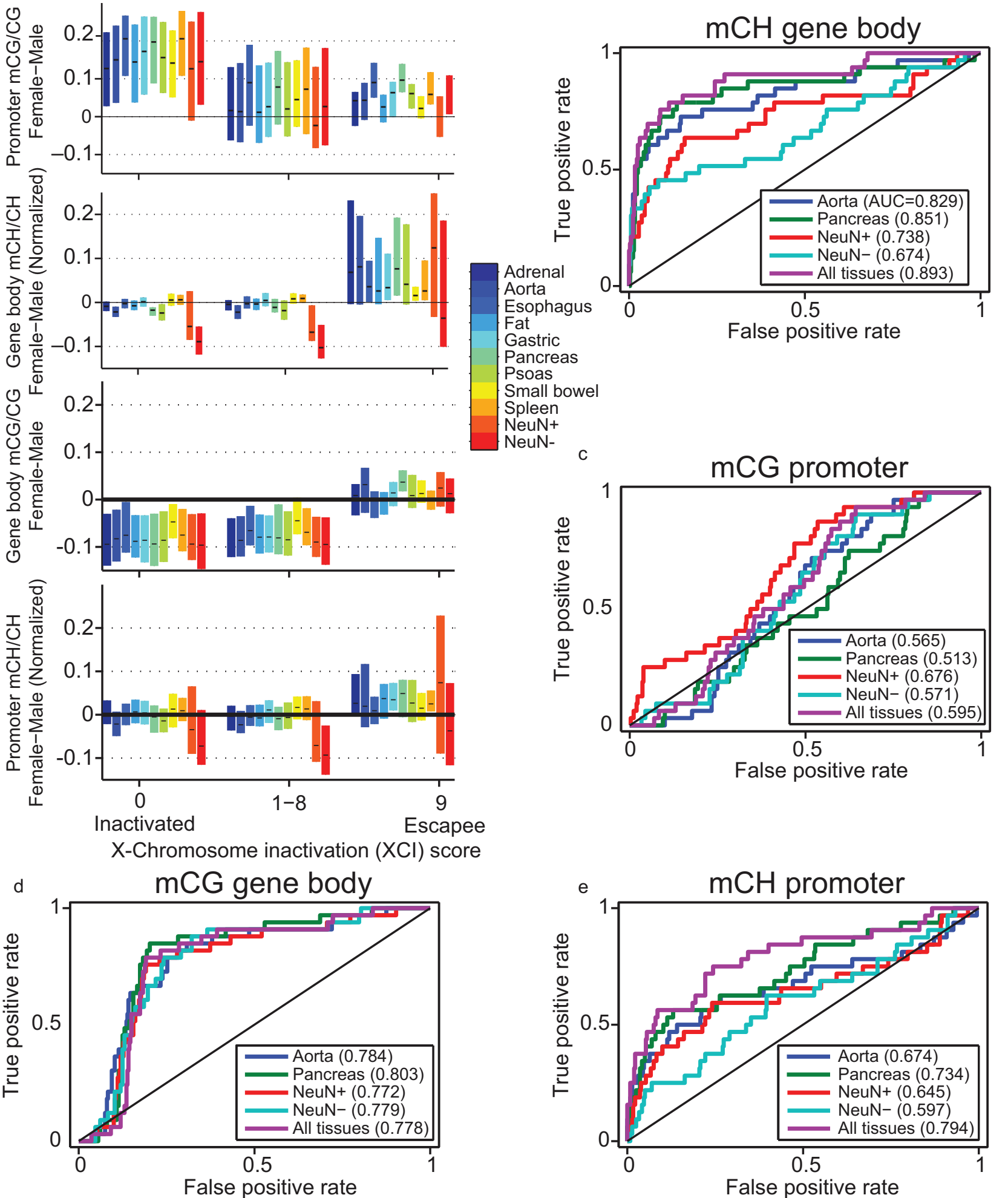

Extended Data Figure 9 $\mid$ X-chromosome inactivation. a, Distributions of promoter CG methylation (mCG) levels (mCG/CG), gene body non-CG methylation $(\mathrm{mCH})$ levels $(\mathrm{mCH} / \mathrm{CH})$, gene body $\mathrm{mCG}$ levels and promoter $\mathrm{mCH}$ levels in genes previously reported to express from only one allele (inactivated) or biallelically (escapee) ${ }^{29}$. Black ticks show median, and bars indicate the twenty-fifth to seventy-fifth percentile range. Genes more prone to escaping inactivation have lower promoter $\mathrm{mCG}$, higher gene body $\mathrm{mCH}$,

higher gene body $\mathrm{mCG}$ and higher promoter $\mathrm{mCH}$ in females. b-e, Discriminability analysis using gender-specific gene body $\mathrm{mCH}(\mathbf{b})$, promoter mCG (c), gene body $\mathrm{mCG}(\mathbf{d})$ and promoter $\mathrm{mCH}(\mathbf{e})$ to predict the escapee status of X-linked genes, respectively. Among them, gene body $\mathrm{mCH}$ is the most predictive feature of X-chromosome inactivation escapees. The discriminability was measured by the area under the curve (AUC) (Supplementary Methods). 
b

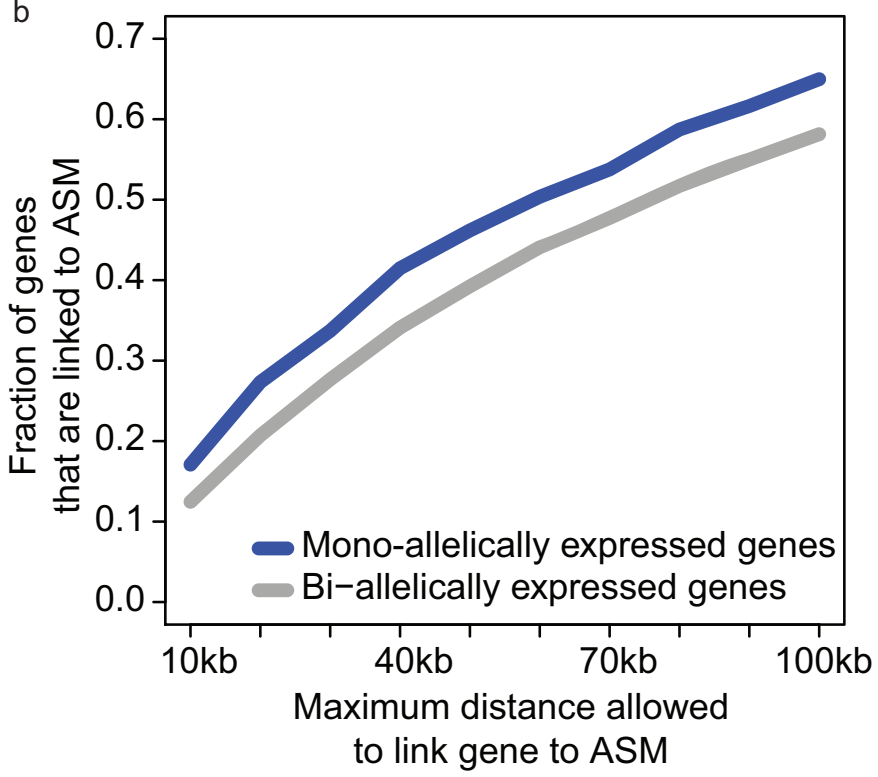
A

Extended Data Figure $10 \mid$ ASM and ASE. a, An example of ASM. Reads that contain a heterozygous SNP (red box) are separated by allele. The number of methylated (reads containing Cs) and unmethylated (reads containing Ts) at adjacent CG sites (black boxes) are tested for differential methylation.

b. Fraction of ASE genes (blue) and bi-allelically expressed genes (grey) that have at least one ASM event within a certain distance. Bi-allelically expressed

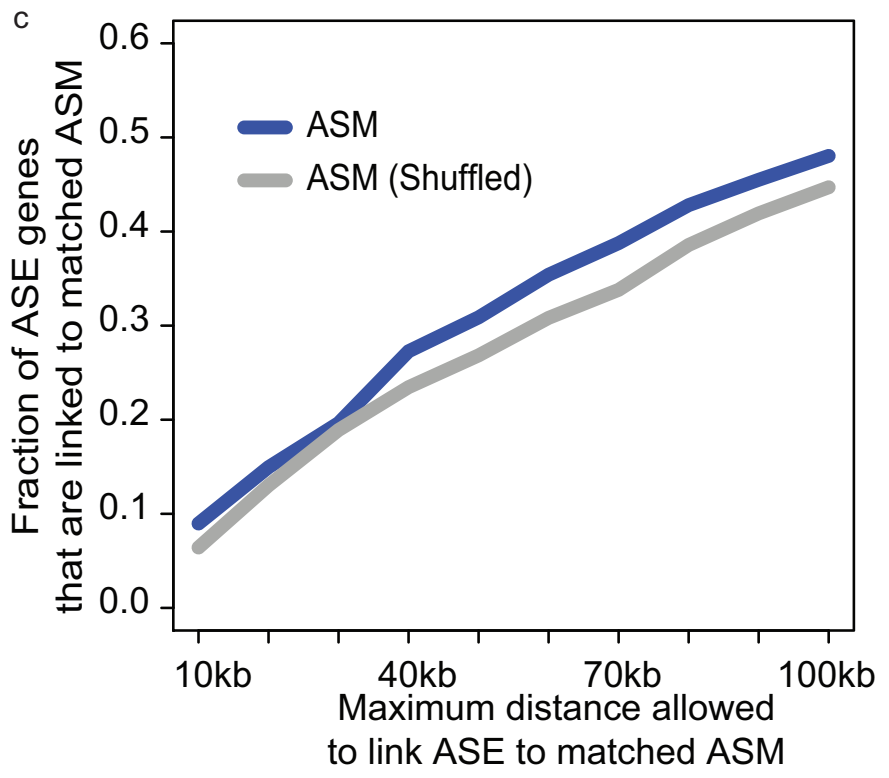

genes were defined as genes that were covered by at least 10 reads and whose $P$ values, given by binomial test for allelic expression, were greater than 0.2 (that is, no significance). c, Fraction of ASE genes that were linked to matched ASM events (blue) and matched ASM events with their locations shuffled (grey). b, c, Aggregated results using samples from triplicate tissues. 


\section{CORRECTIONS \& AMENDMENTS}

CORRIGENDUM

doi:10.1038/nature16179

\section{Corrigendum: Human body} epigenome maps reveal noncanonical DNA methylation

\section{variation}

Matthew D. Schultz, Yupeng He, John W. Whitaker, Manoj Hariharan, Eran A. Mukamel, Danny Leung, Nisha Rajagopal, Joseph R. Nery, Mark A. Urich, Huaming Chen, Shin Lin, Yiing Lin, Inkyung Jung, Anthony D. Schmitt, Siddarth Selvaraj, Bing Ren, Terrence J. Sejnowski, Wei Wang, \& Joseph R. Ecker

Nature 523, 212-216 (2015); doi:10.1038/nature14465

The $y$-axis label in Fig. $3 \mathrm{f}$ of this Letter should read '[mCAC/CAC]/ [mCAG/CAG]' rather than ' $\mathrm{mCAC/CAC]/[mCAC/CAG]'.} \mathrm{In} \mathrm{addi-}$ tion, in the description of Fig. $4 \mathrm{c}$ in the main text, 'tissue' and 'individual' were swapped; it should read, "Of the ASM events that varied, 4.1-7.5\% and 54.5-70.0\% were tissue- and individual-variable, respectively;". These errors have been corrected in the online versions of the paper. 